\title{
Swampland distance conjecture for one-parameter Calabi-Yau threefolds
}

\author{
Abhinav Joshi ${ }^{a}$ and Albrecht Klemm ${ }^{a, b}$ \\ ${ }^{a}$ Bethe Center for Theoretical Physics, \\ University of Bonn, D-53113 Bonn, Germany \\ ${ }^{b}$ Hausdorff Center for Mathematics, \\ University of Bonn, D-53113 Bonn, Germany \\ E-mail: joshi@th.physik.uni-bonn.de, aklemm@th.physik.uni-bonn.de
}

ABSTRACT: We investigate the swampland distance conjecture (SDC) in the complex moduli space of type II compactifications on one-parameter Calabi-Yau threefolds. This class of manifolds contains hundreds of examples and, in particular, a subset of 14 geometries with hypergeometric differential Picard-Fuchs operators. Of the four principal types of singularities that can occur — specified by their limiting mixed Hodge structure - only the $K$-points and the large radius points (or more generally the $M$-points) are at infinite distance and therefore of interest to the SDC. We argue that the conjecture is fulfilled at the $K$ - and the $M$-points, including models with several $M$-points, using explicit calculations in hypergeometric models which contain typical examples of all these degenerations. Together with previous work on the large radius points, this suggests that the SDC is indeed fulfilled for one-parameter Calabi-Yau spaces.

KeYwords: Compactification and String Models, D-branes, Superstring Vacua, Topological Strings

ARXIV EPRINT: 1903.00596 


\section{Contents}

1 Introduction and summary 1

$\begin{array}{lll}2 & \text { Special geometry } & 6\end{array}$

3 One-parameter Calabi-Yau threefolds $\quad 7$

$\begin{array}{lll}3.1 & \text { One-parameter families, monodromies and singularities } & 7\end{array}$

$\begin{array}{lll}3.2 & \text { Metric properties at the critical points } & 10\end{array}$

$\begin{array}{lll}3.3 & \text { Geometric realisation of the hypergeometric cases } & 14\end{array}$

4 Calculation of periods on $\mathcal{M}_{c s}(X) \quad \mathbf{1 5}$

4.1 Symplectic basis at the $M$-point and the monodromy group $\Gamma \subset \operatorname{Sp}(4, \mathbb{Z}) \quad 16$

$\begin{array}{lll}4.2 & \text { Period degeneration near the } C \text {-point } & 17\end{array}$

$\begin{array}{ll}4.3 \text { Around } s \text {-Point }(\psi=0) & 19\end{array}$

5 Global structure of the metric on $\mathcal{M}_{c s}(X) \quad 21$

6 Swampland distance conjecture $\quad 23$

6.1 Light states near the $M$-point 23

6.2 Light states near the $s$-point $\quad 27$

$\begin{array}{lll}6.2 .1 & X_{3,3} & 27\end{array}$

6.2.2 Stability of branes in the $X_{3,3}$ model 28

$\begin{array}{lll}6.2 .3 & X_{2,2,2,2} & 31\end{array}$

6.2.4 Stability of branes in the $X_{2,2,2,2}$ model 32

6.3 Other one parameter hypergeometric systems 34

$\begin{array}{ll}\text { A Meijer G-functions } & 35\end{array}$

\section{Introduction and summary}

Quantum gravity is bound to become important in any consistent UV completion of physics, for which string theory is a good candidate. The criteria which distinguish the vast space of semi-classically consistent low energy theories which admit no consistent UV completion, dubbed the swampland, from the one which possibly has such a completion, called landscape, have been proposed for effective theories, in particular, such with continuous moduli. These criteria are known as the swampland conjectures [1]. While some swampland conjectures such as the weak gravity conjecture are not specific to string theory, it has been argued that they are fulfilled in all known string vacua. This might reflect a certain bias, nevertheless it seems a reasonably general approach to study the border between swampland and landscape starting from within the string theory moduli space. 
In this work, we will examine one of the conjectures called the swampland distance conjecture (SDC) [1]. One starts at a point $p_{0}$ in the interior of the moduli space $\mathcal{M}$ of an effective theory, which in the string theory would be defined by the string-field theory correspondence principle. The conjecture makes a stringent statement about the range of validity of this effective action. It states that the theory at another point $p \in \mathcal{M}$ at a distance $d\left(p, p_{0}\right)$, exhibits an infinite tower of exponentially light states whose mass scales as

$$
m_{p} \sim m_{p_{0}} e^{-\alpha d\left(p, p_{0}\right)}
$$

Here the distance $d\left(p, p_{0}\right)$ is measured with the metric which fixes the kinetic terms of the moduli fields, $m_{p_{0}}$ is the mass of a given state at the point $p_{0}, m_{p}$ is the mass at the point $p$ and $\alpha \in \mathbb{R}_{+}$is an a priori undetermined model dependent parameter. In the refined swampland distance conjecture (RSDC), $\alpha$ is conjectured to be of $\mathcal{O}(1)$ in natural units [2]. The point is that as the distance diverges, an infinite tower of states appears with mass below any finite mass scale used to define the effective action by integrating out the UV modes. This clearly restricts the validity of the effective action to a finite volume in $\mathcal{M}$.

In type IIB compactification on a family of Calabi-Yau threefolds $X_{z}$, the metric for an interesting class of moduli is identified with the Weil-Petersson (WP) metric of the complex structure moduli space $\mathcal{M}_{c s}$ parametrised by the complex moduli $z$. This Kähler metric is given exactly by the period vector $\Pi$ of the Calabi-Yau manifold in an integer symplectic basis. Also, the mass $m_{\mathbf{q}}$ of a BPS 3-brane state with charge vector $\mathbf{q} \in H_{3}(X, \mathbb{Z})$ can be calculated using the periods $\Pi$. Moreover, mirror symmetry allows to extract exact information of the analogous data in the complexified Kähler moduli space $\mathcal{M}_{c k s}$ of type IIA compactifications on the mirror $Y$. Powerful mathematical tools, in particular the Frobenius method for constructing periods, Schmidt's orbit theorem describing the mixed Hodge structure (MHS) at the singular loci $p_{*}$ in $\mathcal{M}_{c s}$ and Bridgeland condition for the stability (BS) of BPS states, strongly restrict the behaviour of the WP metric and the spectrum of (light) BPS states at singular points of infinite distance from the interior of $\mathcal{M}_{c s}$. While they are not strong enough to provide complete arguments to prove the SDC on the complex moduli space in general, the latter makes some interesting and concrete predictions for the period geometry and the BPS spectrum.

It is, therefore, useful to explore SDC in a simple yet variable setting. A very suitable class is that of the one parameter families of Calabi-Yau 3 folds with Betti number $b_{3}=4$, reviewed in [3]. All known Picard-Fuchs operators $\mathcal{L}$, which are of order four and specify, as solutions, four periods of the holomorphic $(3,0)$ form $\Omega$, have been collected in [4]. The form of $\mathcal{L}$ is very much restricted by special geometry and the possible degenerations of geometric periods at singular points $p_{*}$. The latter provides the distinction between points at infinite and finite distance which is of great importance to us. This distinction is encoded in the local monodromy $M \in \mathrm{Sp}(4, \mathbb{Z})$ or more precisely in the limiting mixed Hodge structure (LMHS) as we will explain in section 3.1. There are four principle classes of singularities: $F$-points related to orbifold points, $C$-points are a generalisation ${ }^{1}$ of conifold points, $K$-points are transcendental K3 points and $M$-points which are a generalisation

\footnotetext{
${ }^{1}$ These generalisations can be understood as orbifoldisations.
} 
of the large radius or maximal unipotent monodromy points. Out of these, as shown in section 3.2, only the singularities at $K$ - and $M$-points are of interest, as they are at infinite distance from the interior points on the moduli space.

The test for the SDC on the type IIB compactifications ${ }^{2}$ can be performed as the following. With the classification of the possible infinite distance singularities in [3], it remains to understand the orbifoldisation on top of the $K$ - and $M$-type. Special geometry and the form of LMHS at these singularity allows us to understand the infinite distance property, due to leading logarithmic part of period degeneration. Also, it tells us that there is a 2-dimensional sub-lattice inside the rank four lattice $\Lambda \subset H_{3}(X, \mathbb{Z})$ with an infinite number of primitive ${ }^{3}$ charge vectors $\mathbf{q}$ that could correspond to infinite number of massless BPS 3-brane states. Note that these branes wrap 3-cycles whose relative size vanishes. Their BPS masses $m_{\mathbf{q}}$ become indeed exponentially small as required by (1.1) and with a little more effort one can see more concretely that also the RSDC holds provided these BPS states exist. This second part of the argument is quite successful in establishing that at least the necessary conditions for the SDC hold. It applies to the $K$ - and $M$-points ${ }^{4}$ mentioned above.

We note that even though a rank two charge lattice of light states appears at $K$ - and $M$-points in one moduli cases, all charges in the lattice have symplectic pairing zero so that no Argyres Douglas points with light magnetic and electric charges do occur in this class. Such singular loci can appear if in the type IIA picture a divisor collapses, which is only realisable if there are at least two independent divisor classes and hence at least two moduli, as for instance in the case of the $E$-string in elliptic fibered Calabi-Yau spaces [6]. The swampland conjectures at the corresponding conformal points in F-theory have been studied in [7].

In the decisive part of the argument, one needs to establish that there is an infinite tower of light stable states. In the context of the above-discussed states, one has to check that these infinite primitive charge vectors correspond to stable BPS states in the corresponding region of the moduli space. Powerful techniques like the geodesic web condition [8] that govern the stability question for BPS states in non-gravitational supersymmetric theories are not available for supergravity theories from compact Calabi-Yau spaces. Heuristic arguments using lines of marginal stability where a BPS state of charge $\mathbf{q}$ can decay into two states with linear independent charge vectors $\mathbf{q}_{1}$ and $\mathbf{q}_{2}$, e.g. for arguing the absence of magnetic monopoles of magnetic charge greater than one in the asymptotic free region in [9], are very difficult to apply as the lines of marginal stability become dense.

However, in $N=2$ supergravity in 4 dimensions, there is a Schwinger one loop diagram with external fields from the gravitational sector that generates a coupling $R_{-}^{2} F_{-}^{2 g-2}$ be-

\footnotetext{
${ }^{2}$ We use type IIB language but by mirror symmetry generally the very same techniques translate to type IIA compactifications except for the orphan models in [4].

${ }^{3}$ At the $C$-point, the lattice is 1-dimensional and yields only one primitive vector. A non-primitive vector corresponds to BPS bound states at threshold, which are not stable. Hence there is only one massless particle, to be integrated out to get the Wilsonian effective action.

${ }^{4}$ It has been argued in [5] that it is true for more general LMHS that might occur in multi-parameter Calabi-Yau families.
} 
tween the anti-selfdual parts of the curvature $R_{-}$and the anti-selfdual part of the graviphoton field strength $F_{-}$. For this diagram, at the large radius, the contributions of BPS indices corresponding to $D 2-D 0$ brane bound states can be isolated and are counted by the all genus topological string partition function, which predicts an infinite number of non-zero indices and therefore BPS states. In particular, in the A-model, their vanishing is only enforced by the Castelnuovo conditions, leaving an infinite number of non-vanishing BPS states. These can be calculated using the holomorphic anomaly and the conifold gap condition [10] and have been suggested as light states in the context of the SDC [11]. In the B-model, using general properties of the Picard-Fuchs equations [3] one can conclude that they lead to nontrivial solutions, which at least guarantee an infinite number of genus zero topological string invariants. Moreover, these BPS states have at the large radius points or more generally $M$-points the asymptotic mass behaviour as in (1.1). Mirror symmetry in its stronger form - states that in type IIA string theory compactified to four dimensions on $Y$, the mirror family of $X$, one has the same BPS spectrum from wrapping even cycles in $Y$ than IIB string compactified on $X$ from wrapping 3-cycles. Based on this argument, carried over to the type IIB side, with the corresponding identifications of BPS charges, one can argue that the SDC is valid at the large radius points in type II compactifications. Further, one can also relate it to an exact counting problem that has been solved to a large extent concretely in [10].

The same conclusion about the large radius point has been reached with a similar reasoning in [12], where it is argued that an infinite tower of light Kaluza Klein states appear in the large radius region in type IIA theory. Maybe, one can identify these KK states with some light states on the type IIB side using mirror symmetry. Yet a different argument for an infinite tower of light states was given in $[5,13]$. Here, one assumes the existence of one light stable state and creates an infinite tower of light stable states by a parabolic (infinite order) local monodromy around $p_{*}$, provided that along the loop in $\mathcal{M}$ creating the monodromy, the states building the tower do not cross lines of marginal stability. It is however not clear how the argument could explain the full and intricate spectrum of the dual $D 2-D 0$ brane states calculated in [10], as given a state with fixed $D 2$ charge it only creates an orbit with arbitrary $D 0$ brane charges. Moreover it only establishes that the theory is invariant under monodromy or mutation actions, if this action is accompanied by a relabelling of the charges $\mathbf{q}$ so that the symplectic pairing $\mathbf{q}^{\mathrm{T}} \Sigma \Pi$ is invariant. With this understanding, one does not need to create new states, under this action, to keep the theory invariant.

In any case, none of the arguments stated above work at the $K$-point. The argument of [12] fails because the $K$-points does not correspond at an infinite volume point in type IIA, whereas the argument of $[5,13]$ fails because each primitive state with charge $\mathbf{q}$ creates only a finite orbit under the local monodromy. The latter is the criterion that light states do have to fulfil in order for a local effective action could exist according to [9].

At the $K$-point, all states of the rank two lattice $\Lambda$ are not only in finite orbits, but their symplectic pairing is zero. The first fact does not rule out an effective action a priori according to the argument of [9] and the second fact allows one a Lagrangian description. One has to argue therefore that the primitive vectors in the lattice $\Lambda$ do exist as stable states 
so that an infinite tower of states invalidates the effective action. Our arguments regarding the stability are also not complete, but highly plausible if one follows the reasoning of [14] which states that all states allowed by the Dirac quantisation, which are locally stable, are in the spectrum. To establish the latter point, we analyse the lines of marginal stability for the exponentially light BPS states and show that, for a given charge associated to a state becoming massive at singularity, there is always a finite radius around the $K$ - point in which an infinite number of light states are stable against decay into each other as well as into the above mentioned charge.

This gives good support for the claim that the SDC holds at the $K$-points due to the existence of an infinite tower of stable BPS states. The fact that they are BPS states suggests that at the $K$-points one has a similar interesting counting problem as at large radius that can be related to higher genus string amplitudes captured by the Schwinger loop amplitude, which are known at this points due to the work of [10]. We leave the analysis of this problem for future work.

While the $K$-point is clearly the most challenging from the point of view of establishing the SDC in detail, also, for $M$-point we discover a qualitatively new and interesting phenomenon based on our understanding of the spectrum of the light states. There are one parameter models which have several $M$-points in their moduli space. For example, four quadrics in $\mathbb{P}^{7}$ called $X_{2,2,2,2}$ is a hypergeometric case with two $M$-points in which this feature (of multiple $M$-points) can be studied in an easy setting. One finds that the different leading behaviour of the periods at the second $M$-point relatively to first one can be absorbed into a Kähler transformation, so that the BPS states as captured by the topological string is the same as at the first $M$-point. However, the states that become exponentially light at the second $M$-points and play the role of the $D 2$ and $D 0$ bound states in the Schwinger Loop calculation do not correspond to $D 2$ and $D 0$ branes at the first $M$-point, but rather to a combination involving also $D 6$ and $D 4$ brane charges as seen from the first $M$-point.

This paper is structured as follows: in section 2, we review the principle structure of special geometry, which is a powerful tool for us. In section 3, we review first, in subsection 3.1, the list of one parameter Calabi-Yau manifolds and their degenerations leading to the four types of limiting mixed Hodge structures, up to orbifold actions. We then establish the metric properties from local considerations in subsection 3.2. After a discussion of all hypergeometric cases, we introduce the geometric realisations of the CalabiYau threefolds in subsection 3.3, which serve as our main examples. Section 4 contains the calculations of periods on $\mathcal{M}_{c s}(X)$. We start in subsection 4.1 to fix an integral symplectic basis near the large radius point $(\psi=\infty)$. In subsection 4.2 , we calculate the periods near the conifold $(\psi=1)$ and find the transition matrices. In subsection 4.3 , we write down the periods near the third singular point $(\psi=0)$, using Meijer G-functions, and the transition matrices. With the periods on the whole moduli space and the special geometry, in section 5 , we calculate and plot the metric on $\mathcal{M}_{c s}(X)$ over the moduli space and also give it's limiting behaviour as one approaches the singular points. Section 6 contains the calculations for the SDC. We first contrast the $D 2$ and $D 0$ bound state proposal versus the 
Kaluza Klein proposal in subsection 6.1. Subsection 6.2 discusses, in detail, all the type of singularities that can occur as $\psi \rightarrow 0$ which lead to infinite distance. In particular, we focus on the lattice of exponentially light states at the $K$-point and the second ${ }^{5} M$-point. We first work with the manifold denoted as $X_{3,3}$ in subsection 6.2.1 which has $K$-point at $\psi=0$ and after identifying the candidate stable BPS 3-branes, in section 6.2.2, we study their stability properties analysing their lines of marginal stabilities. We then analyse the $X_{2,2,2,2}$ model in subsection 6.2.3 which has a second $M$-point at $\psi=0$ and identify the candidate states, and in subsection 6.2.4, we analyse the lines of marginal stability. In subsection 6.3, we shortly summarise the findings for the other hypergeometric one parameter models for which $K$-points arise at $\psi=0$, for which, we have done the same analysis as for the key examples. Finally in appendix A, we collect facts about the Meijer G-functions and the Barnes integral method used to make the analysis.

\section{Special geometry}

Let's start by reviewing some facts about the complex structure moduli space, $\mathcal{M}_{c s}(X)$ (or simply $\mathcal{M}_{c s}$ ), which is a special Kähler manifold [15]. Since we will be working with one-parameter spaces, we restrict to $\operatorname{dim}\left(\mathcal{M}_{c s}\right)=1$. On $\mathcal{M}_{c s}$, we can define the Kähler potential as

$$
K(z, \bar{z})=-\log \left(i \int_{X} \Omega(z) \wedge \overline{\Omega(z)}\right)
$$

where $\Omega(z)$ is the non-vanishing $(3,0)$-form on $X$ and $z$ parameterises $\mathcal{M}_{c s}$. This can be used to write down the metric on $\mathcal{M}_{c s}$,

$$
G_{z \bar{z}}=\frac{\partial^{2}}{\partial z \partial \bar{z}} K(z, \bar{z}) .
$$

The metric can be used to calculate the scalar curvature using

$$
R=G^{z \bar{z}} R_{z \bar{z}}=G^{z \bar{z}}\left(-\partial_{z} \bar{\partial}_{z} \log (\operatorname{det} G)\right),
$$

where $G^{z \bar{z}}$ is the inverse of the metric $G_{z \bar{z}}$ and $R_{z \bar{z}}$ is the Ricci tensor. Also, the Yukawa coupling is given $b^{6}$

$$
\kappa_{z z z}=\int_{X} \Omega(z) \wedge \frac{\partial^{3} \Omega(z)}{\partial z \partial z \partial z} .
$$

We now rewrite these quantities using periods. For that, we first choose an integral symplectic basis $A^{I}, B_{I} \in H_{3}(X, \mathbb{Z})$ in the middle homology and a dual basis $\alpha_{I}, \beta^{I} \in$ $H^{3}(X, \mathbb{Z})$ in the middle cohomology with $I=0,1,{ }^{7}$ such that,

$$
A^{I} \cdot B_{J}=-B_{J} \cdot A^{I}=\int_{X} \alpha_{J} \wedge \beta^{I}=-\int_{X} \beta^{I} \wedge \alpha_{J}=\int_{A^{I}} \alpha_{J}=\int_{B_{J}} \beta^{I}=\delta_{J}^{I},
$$

\footnotetext{
${ }^{5}$ Second to the $M$-point at large radius.

${ }^{6}$ These are given just for completeness, we will not be using these at any point in this work.

${ }^{7}$ This is due to the fact that the middle line of Hodge decomposition is: 1111 , summing up to 4 , divided in a pair of 2 .
} 
where $\delta$ is the Kronecker delta and all other pairings vanish. This allows us to define the period vector as

$$
\Pi=\left(\begin{array}{c}
F_{I}(z) \\
X^{I}(z)
\end{array}\right)=\left(\begin{array}{c}
\int_{B_{I}} \Omega(z) \\
\int_{A^{I}} \Omega(z)
\end{array}\right) .
$$

Inverting this relation, we can write

$$
\Omega(z)=X^{I}(z) \alpha_{I}-F_{I}(z) \beta^{I} .
$$

Inserting this in equation (2.1), we find

$$
\begin{aligned}
K(z, \bar{z}) & =-\log \left(i\left(\overline{X^{I}(z)} F_{I}(z)-X^{I}(z) \overline{F_{I}(z)}\right)\right) \\
& =-\log \left(-i \Pi(z)^{\dagger} \Sigma \Pi(z)\right)
\end{aligned}
$$

where

$$
\Sigma=\left(\begin{array}{cc}
0 & \operatorname{Id}_{2 \times 2} \\
-\operatorname{Id}_{2 \times 2} & 0
\end{array}\right) .
$$

Therefore, once we have the periods, we can calculate the Kähler potential using equation (2.8) and then find the metric using equation (2.2).

\section{One-parameter Calabi-Yau threefolds}

In this section, we will describe the moduli geometry of complex families of Calabi-Yau threefolds and recall the classification and properties of their singular fibers.

\subsection{One-parameter families, monodromies and singularities}

It is well known that the most useful classifications of these special fibers is provided by the monodromies of the periods of the holomorphic 3-form, which can be calculated from the solutions of the Picard-Fuchs operator $\mathcal{L}$ once an integral symplectic basis is provided. The monodromies in this basis respect the symplectic pairing (2.5), which renders them in $\operatorname{Sp}(4, \mathbb{Z})$.

The coarsest classification of these types of monodromies $M$ is given by the finite order $k$ of the branch running into ${ }^{8} p_{*}$ and the least $n \leq \operatorname{dim}_{\mathbb{C}}(X)$ determining the degree of nilpotency of $M$, i.e.

$$
\left(M^{k}-\mathbf{1}\right)^{n+1}=0 .
$$

It is convenient to apply a multiplicative Jordan-Chevalley decomposition to $M$ and split it as

$$
M=U S,
$$

where $U$ and $S$ is a commuting pair of unipotent and semi simple matrices respectively. Special geometry and the fact that only regular singular points should occur restricts the form of possible differential operators $\mathcal{L}$. Moreover if one assumes a point of maximal

\footnotetext{
${ }^{8}$ Locally one can always choose multi-covering variables $\psi=z^{\frac{1}{k}}$ to remove the branch cut. However, globally convenient variables have the branch cuts.
} 
unipotent monodromy (MUM), given by $k=1$ and $n=3$, then the integrality of the mirror map as well as of the genus zero BPS numbers yields further constraints. In particular for a fixed number of singularities and apparent singularities one can provide classifications (see [4] and [3] for a recent review). For example there are exactly 14 hypergeometric cases with three singularities including a MUM point and no apparent singularities [16]. Thirteen are realised geometrically by generically smooth hyper-surfaces in weighted projective spaces [17-19] or complete intersections in projective spaces [20] or weighted projective spaces [21]. A summary of these geometrical cases including the behaviour of all genus potentials at the singularities can be found in [10].

The list [4] contains however much more general examples that have unsuspected properties, like models with multiple MUM points leading to topological different mirrors or geometric examples that have no MUM point, so called orphans, leading to families with no geometric mirror.

There are finer types of invariants than the $k$ and $n$ in (3.1) associated to the singular points $p_{*}$, in particular using the nilpotent operator

$$
N=\log (U),
$$

one can define in addition to the $F^{p}=\oplus_{i \geq p} H^{i, 3-i}(X)$ filtration, a second $W_{*}$ filtration for the limiting Hodge structure that refines nilpotency index $n$ into the information in the limiting mixed Hodge structure (LMHS). In the following, we mention some basic definitions ${ }^{9}$ that leads to the Hodge diamonds shown below. The $W_{*}$ filtration $W_{0} \subset W_{1} \subset$ $\ldots \subset W_{2 n-1} \subset W_{2 n}=H_{\text {lim }}^{3}\left(X_{s}\right)$ at the singular point $s$ is given by the action of $N$ as $W_{0}=$ $\operatorname{Im}\left(N^{n}\right), W_{1}=\operatorname{Im}\left(N^{n-1}\right) \cap \operatorname{ker}(N), W_{2}=\operatorname{Im}\left(N^{n-2}\right) \cap \operatorname{ker}(N)+\operatorname{Im}\left(N^{n-1}\right) \cap \operatorname{ker}\left(N^{2}\right), \cdots$, $W_{2 n-1}=\operatorname{ker}\left(N^{n}\right)$. Note that $N\left(W_{k}\right) \subset W_{k-2}$. On the quotient spaces $\mathrm{Gr}_{k}=W_{k} / W_{k-2}$, the $N^{k}$ induce an isomorphism $N^{k}: \mathrm{Gr}_{n+k} \rightarrow \mathrm{Gr}_{n-k}$ and $F_{\lim }^{*}$ defines a rank $k$ Hodge structure on the spaces $\mathrm{Gr}_{k}$. The Hodge diamonds of the LMHS shows these $\mathrm{Gr}_{k}$, with $k=0, \ldots, 2 n-1$ from bottom to top and for each $\mathrm{Gr}_{k}$, the Hodge decomposition. From above it follows that $N$ acts by a shift of two rows and the original entries can only be shifted up or down along SW-NE lines.

For one-parameter CY families, the LMHS is, to a large extent, already captured by the local exponents $\left(a_{1}, a_{2}, a_{3}, a_{4}\right)$ with $a_{i} \in \mathbb{Q}$ at the singular point $p_{*}$ at $x_{*}=0$. These exponents are determined by solving for $a$ with multiplicities in $\mathcal{L}\left(x_{*}\right) x_{*}^{a} \sum_{k=0}^{\infty} c_{k} x_{*}^{k}=0$, where $x_{*}$ is a local complex structure coordinate. They are summarised in the columns of the Riemann Symbol associated to $\mathcal{L}$, see e.g. (4.2) for the hypergeometric cases. For this reason, it is convenient to index the $\mathcal{L}$ on [4] by their local exponents. The most well known singularities, the MUM points and the conifold points with local exponents $(0,0,0,0)$ and $(0,1,1,2)$ respectively $(4.2)$ occur in thousands of different $\mathcal{L}$ often with higher multiplicity then one.

However, in total there are only 123 different local exponents in [4]. For 57 of them, the difference between different $a_{i}$ is either zero or a non-vanishing rational number (not

\footnotetext{
${ }^{9}$ See [3] for an explanation in the one-parameter case and [22] for the general theory for physicist and further references.
} 
in $\mathbb{Z}$ ). In this case one can infer the local types immediately from the local exponents and these singularities fall into four principal types.

- $F$ : $M$ is of finite order $k$ if the exponents are all different $(a, b, c, d)$. The LMHS diamond is

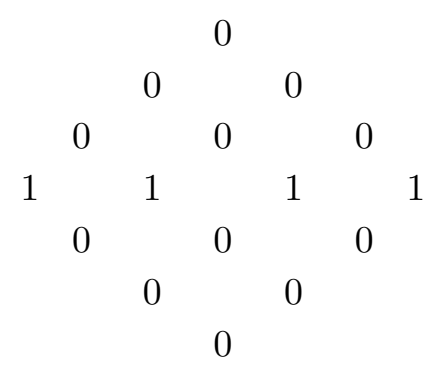

- $C: M$ has one $2 \times 2$ Jordan block of infinite order if the exponent structure is $(a, b, b, c)$, and the LMHS diamond is

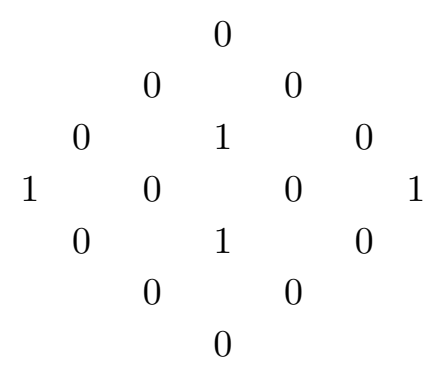

- $K$ : $M$ has two such Jordan blocks if the exponent structure is $(a, a, b, b)$ and the LMHS diamond is

$\begin{array}{cccccccc} & & & 0 & & & \\ & & 0 & & 0 & & \\ & & & & 0 & & 1 & \\ 0 & & 0 & & 0 & & 0 \\ & 1 & & 0 & & 1 & \\ & & & & & & & \\ & & 0 & & 0 & & \\ & & & 0 & & & & \end{array}$

- $M: M$ has a $4 \times 4$ Jordan block of infinite order if the exponents are all equal, i.e. $(a, a, a, a)$ and the LMHS diamond is

$\begin{array}{cccccccc} & & & 1 & & & \\ & & 0 & & 0 & & \\ & 0 & & 1 & & 0 & \\ 0 & & 0 & & 0 & & 0 \\ & 0 & & 1 & & 0 & \\ & & 0 & & 0 & & \\ & & & 1 & & & \end{array}$


We note that the singular points of type $F$ and $C$ are at finite distance as measured with the WP metric, while type $K$ and $M$ are at infinite distance. This follows simply from the logarithmic structure of the solutions associated with the Jordan block of infinite order as we will see in the next subsection. In all cases, $k$ in equation (3.1) is the LCM of the denominator of the $a_{i}$. The 66 remaining exponents have integer differences between the different $a_{i}$. They exhibit the same structure of the local exponents above and the same Jordan blocks can occur but the question if they actually occur requires next to leading order study of the actual solutions to $\mathcal{L}$. For example, the conifold is indeed of type $C$. Note that the pattern $(a, b, b, b)$ with a $3 \times 3$ Jordan block of finite or infinite order can be excluded by special geometry.

\subsection{Metric properties at the critical points}

In the following, we would like to comment on the distance to the different types of singular points that can occur. We give an overview of the general structure, while explicit calculation for concrete models can be found in later sections. For the 14 hypergeometric families with $\psi$ parametrising the moduli space, table 1 shows the type of the singular point at $\psi=0,{ }^{10}$ the name of the threefold and the exponents near $\psi=0$ in the first three columns. The fourth column has $\mu$ which is used in the next sections and it appears in the Picard-Fuchs equation. The fifth and sixth column contains topological numbers ${ }^{11}$ $\kappa=\int_{X} \omega^{3}=D^{3}$ and $c_{2} \cdot D=\int_{X} c_{2}(T X) \wedge \omega$ required for finding periods in symplectic basis and most importantly, column seven has the metric structure as one approaches the singular point (at $\psi=0$ ) and the eighth column has the distance measured from a nonsingular point to $\psi=0$.

First, let us show that the $M$ - and $K$-points are at infinite distance and all others are at finite distance. For this we will be using the Schmidt's orbit theorem [24] which allows us to separate the part responsible for monodromy transformation and a monodromy invariant power series. To simplify the analysis, we perform coordinate transformation $\tilde{x}=x$ where $\tilde{x}$ is the local coordinate and $k$ is the LCM of denominator of $a_{i}$. This transformation gets rid of the semi simple part of the monodromy and allows us to write the period as

$$
\Pi=\exp \left(\frac{1}{2 \pi i} \log (x) N\right) \mathbf{A}(x)
$$

where $\mathbf{A}(x)$ is a holomorphic function which can be expanded as

$$
\mathbf{A}(x)=\mathbf{a}_{0}+\mathbf{a}_{1} x+\mathbf{a}_{2} x^{2}+\ldots
$$

and $N=\log (U)$ where $U$ is the unipotent part of the monodromy matrix obtained by the transformation $\Pi(x) \rightarrow \Pi\left(e^{2 \pi i} x\right)=U \Pi(x)$. Since $U$ is unipotent, $N$ is nilpotent meaning that $N^{i-1} \neq 0$ and $N^{i}=0$ for some $i \in \mathbb{Z}^{+}$. For $F$-points, $i=1$, for $C$ - and $K$-points, $i=2$ and for $M$-points, $i=4$. Before writing the Kähler potential, we define another coordinate $t=\frac{1}{2 \pi i} \log (x)=v+i y$ (not the mirror map).

\footnotetext{
${ }^{10}$ See section 3.3 for notation of manifold and relation of $\psi$ and $z$, where $z$ appears in the Picard-Fuchs equation (4.1).

${ }^{11}$ The calculation for these topological numbers can be found in [23].
} 


\begin{tabular}{|c|c|c|c|c|c|c|c|}
\hline Type & Threefold & $\left(a_{1}, a_{2}, a_{3}, a_{4}\right)$ & $\mu$ & $\kappa$ & $c_{2} \cdot D$ & Metric & Dist. \\
\hline$M$ & $X_{2,2,2,2}\left(1^{8}\right)$ & $\left(\frac{1}{2}, \frac{1}{2}, \frac{1}{2}, \frac{1}{2}\right)$ & $2^{8}$ & 16 & 64 & $\frac{3}{4|\psi|^{2} \log ^{2}(|\psi|)}$ & $\infty$ \\
\hline$F$ & $X_{4,3}\left(1^{5} 2\right)$ & $\left(\frac{1}{4}, \frac{1}{3}, \frac{2}{3}, \frac{3}{4}\right)$ & $2^{6} 3^{3}$ & 6 & 48 & $\frac{3^{3} \Gamma\left(\frac{1}{3}\right)^{6} \Gamma\left(\frac{3}{4}\right)^{4}}{2 \pi \Gamma\left(\frac{1}{4}\right)^{8}|\psi|}$ & $<\infty$ \\
\hline$C$ & $X_{4,2}\left(1^{6}\right)$ & $\left(\frac{1}{4}, \frac{1}{2}, \frac{1}{2}, \frac{3}{4}\right)$ & $2^{10}$ & 8 & 56 & $-\frac{23^{3} \Gamma\left(\frac{3}{4}\right)^{12}|\psi| \log (|\psi|)}{\pi^{6}}$ & $<\infty$ \\
\hline$F$ & $X_{5}\left(1^{5}\right)$ & $\left(\frac{1}{5}, \frac{2}{5}, \frac{3}{5}, \frac{4}{5}\right)$ & $5^{5}$ & 5 & 50 & $\frac{5^{2} \Gamma\left(\frac{2}{5}\right)^{5} \Gamma\left(\frac{4}{5}\right)^{5}}{\Gamma\left(\frac{1}{5}\right)^{5} \Gamma\left(\frac{3}{5}\right)^{5}}$ & $<\infty$ \\
\hline$K$ & $X_{3,3}\left(1^{6}\right)$ & $\left(\frac{1}{3}, \frac{1}{3}, \frac{2}{3}, \frac{2}{3}\right)$ & $3^{6}$ & 9 & 54 & $\frac{1}{4|\psi|^{2} \log ^{2}(|\psi|)}$ & $\infty$ \\
\hline$K$ & $X_{4,4}\left(1^{4} 2^{2}\right)$ & $\left(\frac{1}{4}, \frac{1}{4}, \frac{3}{4}, \frac{3}{4}\right)$ & $2^{12}$ & 4 & 40 & $\frac{1}{4|\psi|^{2} \log ^{2}(|\psi|)}$ & $\infty$ \\
\hline$C$ & $X_{3,2,2}\left(1^{7}\right)$ & $\left(\frac{1}{3}, \frac{1}{2}, \frac{1}{2}, \frac{2}{3}\right)$ & $2^{4} 3^{3}$ & 12 & 60 & $-\frac{7^{3} \Gamma\left(\frac{5}{6}\right)^{9}|\psi|^{1 / 3} \log (|\psi|)}{2^{8 / 3} \pi^{9 / 2}}$ & $<\infty$ \\
\hline$C$ & $X_{6,2}\left(1^{5} 3\right)$ & $\left(\frac{1}{6}, \frac{1}{2}, \frac{1}{2}, \frac{5}{6}\right)$ & $2^{8} 3^{3}$ & 4 & 52 & $-\frac{3^{3} \Gamma\left(\frac{5}{6}\right)^{9}|\psi|^{2} \log (|\psi|)}{2^{1 / 3} \pi^{9 / 2}}$ & $<\infty$ \\
\hline$F$ & $X_{6}\left(1^{4} 2\right)$ & $\left(\frac{1}{6}, \frac{1}{3}, \frac{2}{3}, \frac{5}{6}\right)$ & $2^{4} 3^{6}$ & 3 & 42 & $\frac{100 \sqrt[3]{2} \pi^{7 / 2}}{\sqrt{3} \Gamma\left(\frac{1}{6}\right)^{5} \Gamma\left(\frac{1}{3}\right)^{2}|\psi|^{1 / 3}}$ & $<\infty$ \\
\hline$F$ & $X_{8}\left(1^{4} 4\right)$ & $\left(\frac{1}{8}, \frac{3}{8}, \frac{5}{8}, \frac{7}{8}\right)$ & $2^{16}$ & 2 & 44 & $0.0815626|\psi|^{1 / 2}$ & $<\infty$ \\
\hline F & $X_{6,4}\left(1^{3} 2^{2} 3\right)$ & $\left(\frac{1}{6}, \frac{1}{4}, \frac{3}{4}, \frac{5}{6}\right)$ & $2^{10} 3^{3}$ & 12 & 32 & $\frac{0.0103506}{|\psi|}$ & $<\infty$ \\
\hline$F$ & $X_{10}\left(1^{3} 2,5\right)$ & $\left(\frac{1}{10}, \frac{3}{10}, \frac{7}{10}, \frac{9}{10}\right)$ & $2^{8} 5^{5}$ & 1 & 34 & 0.0424127 & $<\infty$ \\
\hline$K$ & $X_{6,6}\left(1^{2} 2^{2} 3^{2}\right)$ & $\left(\frac{1}{6}, \frac{1}{6}, \frac{5}{6}, \frac{5}{6}\right)$ & $2^{8} 3^{6}$ & 1 & 22 & $\frac{1}{4|\psi|^{2} \log ^{2}(|\psi|)}$ & $\infty$ \\
\hline$F$ & $X_{2,12}\left(1^{4} 4,6\right)$ & $\left(\frac{1}{12}, \frac{5}{12}, \frac{7}{12}, \frac{11}{12}\right)$ & $2^{12} 3^{6}$ & 1 & 46 & $\frac{9 \Gamma\left(\frac{5}{6}\right)^{6}|\psi|^{2}}{8 \sqrt[3]{2} \pi^{3}}$ & $<\infty$ \\
\hline
\end{tabular}

Table 1. Showing the metric as $\psi \rightarrow 0$ on moduli space of 14 hypergeometric one parameter Calabi-Yau manifolds. Also, the distance to $\psi=0$.

The Kähler potential can then be written as

$$
\begin{aligned}
& K(t, \bar{t})=-\log \left(-i \mathbf{A}(t)^{\dagger} e^{\bar{t} N^{\mathrm{T}}} \Sigma e^{t N} \mathbf{A}(t)\right) \\
& =-\log \left(-i \mathbf{A}(t)^{\dagger} \Sigma e^{(t-\bar{t}) N} \mathbf{A}(t)\right) \\
& =-\log \left(-i\left(\mathbf{a}_{0}^{\dagger}+\mathbf{a}_{1}^{\dagger} e^{-2 \pi i \bar{t}}+\ldots\right) \Sigma e^{(t-\bar{t}) N}\left(\mathbf{a}_{0}+\mathbf{a}_{1} e^{2 \pi i t}+\ldots\right)\right) \text {, }
\end{aligned}
$$

where in second line, we have used the relation $N^{\mathrm{T}} \Sigma=-\Sigma N$ which can be deduced using $U^{\mathrm{T}} \Sigma U=\Sigma$. For the periods near $x=0$ or $t=i \infty$, one finds that $\mathbf{a}_{i}=0$ for 
$i<m \equiv \min \left\{k a_{1}, k a_{2}, k a_{3}, k a_{4}\right\}$, which can be understood from the local solutions around the singular point. Hence, we find that

$$
\begin{aligned}
K(t, \bar{t}) & =-\log \left(-i \mathbf{a}_{m}^{\dagger} \Sigma e^{2 i y N} \mathbf{a}_{m} e^{-4 \pi m y}+\mathcal{O}\left(e^{-4 \pi m y-2 \pi y}\right)\right) \\
& =4 \pi m y-\log \left(-i \mathbf{a}_{m}^{\dagger} \Sigma e^{2 i y N} \mathbf{a}_{m}+\mathcal{O}\left(e^{-2 \pi y}\right)\right) .
\end{aligned}
$$

The first term above can be removed using a Kähler transformation $K(t, \bar{t}) \rightarrow K(t, \bar{t})-$ $f(t)-\bar{f}(\bar{t})$ with $f(t)=-2 \pi m i t$ and $\bar{f}(\bar{t})=2 \pi m i \bar{t}$. Therefore, we get

$$
\begin{aligned}
K(t, \bar{t}) & =-\log \left(-i \mathbf{a}_{m}^{\dagger} \Sigma e^{2 i y N} \mathbf{a}_{m}+\mathcal{O}\left(e^{-2 \pi y}\right)\right) \\
& =-\log (p(y)+\mathbf{h})
\end{aligned}
$$

where $p(y)$ is a polynomial of degree $d$ since $N$ is nilpotent. Note that for $y \rightarrow \infty$, the $\mathbf{h}=\mathcal{O}\left(e^{-2 \pi y}\right)$ term and all of it's partial derivatives are exponentially suppressed. Using this, one can readily calculate the metric as

$$
\begin{aligned}
G=\partial_{t} \bar{\partial}_{\bar{t}} K(t, \bar{t}) & =\frac{1}{4}\left(\partial_{y} \partial_{y}+\partial_{v} \partial_{v}\right) K(v, y) \\
& =-\frac{1}{4} \frac{\left(p(y)^{\prime \prime}+\mathbf{h}\right)(p(y)+\mathbf{h})-\left(p(y)^{\prime}+\mathbf{h}\right)^{2}}{(p(y)+\mathbf{h})^{2}} \\
& =-\frac{1}{4} \frac{p(y)^{\prime \prime} p(y)-p(y)^{\prime 2}+\mathbf{h}}{p^{2}+\mathbf{h}} \sim \frac{1}{4} \frac{p^{\prime 2}-p^{\prime \prime} p}{p^{2}}+\mathbf{h} \\
& \sim \frac{1}{4} \frac{d^{2}-d(d-1)}{y^{2}}+\mathbf{h}=\frac{d}{y^{2}}+\mathbf{h} .
\end{aligned}
$$

Therefore, for $d=0$, we get finite distance on integrating the metric distance from some non singular point to $y=\infty$, whereas, for $d>0$, the distance diverges logarithmically as $y \rightarrow \infty$. As noticed in [25], one can conclude that the degree of the polynomial $d=$ $\operatorname{deg} p(y)=\max \left\{l, N^{l} \mathbf{a}_{m} \neq 0\right\}$.

In the following, we will be using Frobenius basis instead of symplectic basis. Let's denote the Frobenius basis by $\tilde{\Pi}$ and the transformation matrix by $T$ such that

$$
\Pi=T \tilde{\Pi}=T \exp \left(\frac{1}{2 \pi i} \log (x) \tilde{N}\right) \tilde{\mathbf{A}}(x),
$$

which, using equation (3.4), can be rewritten as

$$
\begin{aligned}
\exp \left(\frac{1}{2 \pi i} \log (x) N\right) \mathbf{A}(x) & =T \exp \left(\frac{1}{2 \pi i} \log (x) \tilde{N}\right) \tilde{\mathbf{A}}(x) \\
\Longrightarrow \exp \left(\frac{1}{2 \pi i} \log (x) N\right) \mathbf{A}(x) & =\exp \left(\frac{1}{2 \pi i} \log (x) T \tilde{N} T^{-1}\right) T \tilde{\mathbf{A}}(x) .
\end{aligned}
$$

On comparing the two sides, we can read off that

$$
N=T \tilde{N} T^{-1}, \quad \mathbf{A}(x)=T \tilde{\mathbf{A}}(x)
$$

and hence, the statement for the degree $d$ translates to $d=\operatorname{deg} p(y)=\max \left\{l, \tilde{N}^{l} \tilde{\mathbf{a}}_{m} \neq 0\right\}$. 
Let's look at each type of singular point:

- $F$-point $(a, b, c, d)$ : for this type, we know that $\tilde{N}=0(N=0)$ from above, hence $d=0$, making it a finite distance point.

- $C$-point $(a, b, b, c)$ : for this type, with $a<b<c$, the structure of exponents locally allow us to write the periods in Frobenius basis as

$$
\tilde{\Pi}=\left(\begin{array}{c}
\nu_{0}(x) \\
\nu_{0}(x) \log (x)+s_{0}(x) \\
s_{1}(x) \\
s_{2}(x)
\end{array}\right) \text {, with } \tilde{\mathbf{a}}_{m}=\left(\begin{array}{l}
0 \\
0 \\
\alpha \\
0
\end{array}\right) .
$$

Here $\nu_{0}(x)$ and $s_{0}(x)$ are power series with leading term of order $x^{k b}, s_{1}(x)$ and $s_{2}(x)$ are power series with leading term of order $x^{k a}$ and $x^{k c}$ respectively and $\alpha \in \mathbb{C}$. Note that here $m=k a$. In this basis, the unipotent part of the monodromy matrix and it's logarithm can be written as

$$
\tilde{U}=\left(\begin{array}{cccc}
1 & 0 & 0 & 0 \\
2 \pi i & 1 & 0 & 0 \\
0 & 0 & 1 & 0 \\
0 & 0 & 0 & 1
\end{array}\right), \quad \tilde{N}=\log (\tilde{U})=\left(\begin{array}{cccc}
0 & 0 & 0 & 0 \\
2 i \pi & 0 & 0 & 0 \\
0 & 0 & 0 & 0 \\
0 & 0 & 0 & 0
\end{array}\right)
$$

Note that this monodromy is due to the logarithmic term in the period vector $\tilde{\Pi}$. The contribution to the monodromy from power series due to the branch cuts in the power series shows up in semi-simple part and is irrelevant for the argument about the metric. Clearly, for the above $\tilde{N} \tilde{\mathbf{a}}_{m}=0$. Hence, $d=0$, making the $C$ point a finite distance point.

- $K$-point $(a, a, b, b)$ : for this point, with $a<b$, the exponents imply that the solutions are given by

$$
\tilde{\Pi}=\left(\begin{array}{c}
\nu_{0}(x) \\
\nu_{0}(x) \log (x)+s_{0}(x) \\
\nu_{1}(x) \\
\nu_{1}(x) \log (x)+s_{1}(x)
\end{array}\right), \text { with } \tilde{\mathbf{a}}_{m}=\left(\begin{array}{c}
\alpha \\
\alpha^{\prime} \\
0 \\
0
\end{array}\right)
$$

where $\nu_{0}(x)$ and $s_{0}(x)$ are power series with leading term of order $x^{a k}, \nu_{1}(x)$ and $s_{1}(x)$ have leading term order $x^{b k}$ and $\alpha, \alpha^{\prime} \in \mathbb{C}$. Here, $m=a k$. Again, the unipotent part of the monodromy and it's logarithm will be

$$
\tilde{U}=\left(\begin{array}{cccc}
1 & 0 & 0 & 0 \\
2 \pi i & 1 & 0 & 0 \\
0 & 0 & 1 & 0 \\
0 & 0 & 2 \pi i & 1
\end{array}\right), \quad \tilde{N}=\log (\tilde{U})=\left(\begin{array}{cccc}
0 & 0 & 0 & 0 \\
2 i \pi & 0 & 0 & 0 \\
0 & 0 & 0 & 0 \\
0 & 0 & 2 i \pi & 0
\end{array}\right) \text {. }
$$


Note that now

$$
\tilde{N} \tilde{\mathbf{a}}_{m}=\left(\begin{array}{c}
0 \\
2 \pi i \alpha \\
0 \\
0
\end{array}\right), \quad \text { and } \quad \tilde{N}^{2} \tilde{\mathbf{a}}_{m}=0 .
$$

Hence, we can conclude that $d=1$ for $K$ points, making it an infinite distance point.

- $M$-point $(a, a, a, a)$ : here, exponents lead us to the following period vector

$$
\tilde{\Pi}=\left(\begin{array}{c}
\nu_{0}(x) \\
\nu_{0}(x) \log (x)+s_{0}(x) \\
\nu_{0}(x) \log ^{2}(x)+2 s_{0}(x) \log (x)+s_{1}(x) \\
\nu_{0}(x) \log ^{3}(x)+3 s_{0}(x) \log ^{2}(x)+3 s_{1}(x) \log (x)+s_{2}(x)
\end{array}\right) \text {, with } \tilde{\mathbf{a}}_{m}=\left(\begin{array}{c}
\alpha \\
\alpha^{\prime} \\
\alpha^{\prime \prime} \\
\alpha^{\prime \prime \prime}
\end{array}\right),
$$

where $\nu_{0}(x), s_{0}(x), s_{1}(x)$ and $s_{2} x$ are power series with leading term of order $x^{a k}$ and $\alpha, \alpha^{\prime}, \alpha^{\prime \prime}, \alpha^{\prime \prime \prime} \in \mathbf{C}$, also $m=a k$. The period $\tilde{\Pi}$ can be used to calculate the unipotent component of monodromy,

$$
\tilde{U}=\left(\begin{array}{cccc}
1 & 0 & 0 & 0 \\
2 i \pi & 1 & 0 & 0 \\
-4 \pi^{2} & 4 i \pi & 1 & 0 \\
-8 i \pi^{3} & -12 \pi^{2} & 6 i \pi & 1
\end{array}\right), \quad \tilde{N}=\log (\tilde{U})=\left(\begin{array}{cccc}
0 & 0 & 0 & 0 \\
2 i \pi & 0 & 0 & 0 \\
0 & 4 i \pi & 0 & 0 \\
0 & 0 & 6 i \pi & 0
\end{array}\right)
$$

And using the $\tilde{N}$ and $\tilde{\mathbf{a}}_{m}$, we can calculate

$$
\tilde{N}^{3} \tilde{\mathbf{a}}_{m}=\left(\begin{array}{c}
0 \\
0 \\
0 \\
-48 \pi^{3} i \alpha
\end{array}\right), \quad \text { and } \quad \tilde{N}^{4} \tilde{\mathbf{a}}_{m}=0
$$

where the last part is expected since $\tilde{N}^{4}=0$ for $M$ point. Hence, for $M$ point, $d=3$ making it an infinite distance point.

\subsection{Geometric realisation of the hypergeometric cases}

In this section, we will state the Calabi-Yau threefolds which are of interest to us. We start with a set of $n-3$ homogeneous polynomials of degree $d_{1}, \ldots, d_{n-3}$ in the complex weighted projective space $\mathbb{P}^{n}\left(w_{1}, \ldots, w_{n+1}\right)$. A non-zero complete intersection, $Y$, of the zero locus of these polynomials will be a 3 dimensional complex manifold. For this manifold to be CalabiYau, we need its first Chern class to vanish, which translates to the following condition

$$
n+1=\sum_{k=1}^{n-3} d_{k} .
$$

In the following, we will study in detail the threefolds in projective space with all weights equal to one, i.e. $w_{i}=1$. It turns out that this subset in itself gives us a rich class 
of examples and has all the types of singular points mentioned above allowing us to draw general qualitative conclusions.

Since $d_{k}=1$ corresponds to a linear subspace of $\mathbb{P}^{n}$, which is $\mathbb{P}^{n-1}$, we restrict to $d_{k}>1$. This leads to only five possibilities:

- One quintic in $\mathbb{P}^{4}$,

- one cubic and two quadratics in $\mathbb{P}^{6}$,

- one quartic and one quadratic in $\mathbb{P}^{5}$,

- two cubics in $\mathbb{P}^{5}$, and

- four quadratics in $\mathbb{P}^{7}$.

As mentioned before, we are going to work with the mirrors of these manifolds. Let's denote the mirror of a given complete intersection by $X_{d_{1}, \ldots, d_{n-3}}\left(w_{1}, \ldots, w_{n+1}\right) .{ }^{12}$ Hence, mirrors of the list above can be conveniently written as $X_{5}\left(1^{5}\right), X_{3,2,2}\left(1^{7}\right), X_{4,2}\left(1^{6}\right), X_{3,3}\left(1^{6}\right)$ and $X_{2,2,2,2}\left(1^{8}\right) \cdot{ }^{13}$ The mirror of the quintic $X_{5}$ can be calculated by quotient action on the general quintic by the group $\mathbb{Z}_{5}^{3}$ [26]. For $X_{3,3}$, the mirror was correctly guessed in [27]. In [28], the authors find the mirror for the rest of the cases. To parameterise the moduli space, we will switch between two coordinate systems depending on convenience. These are $z$ and $\psi$ related as

$$
z=\frac{1}{\mu \psi^{n+1}}, \quad \text { for } \quad 0 \leq \operatorname{Arg}(z)<2 \pi, \quad 0 \leq \operatorname{Arg}(\psi)<\frac{2 \pi}{n+1} .
$$

\section{Calculation of periods on $\mathcal{M}_{c s}(X)$}

The period vector $\Pi$ is the solution of the following Picard-Fuchs equation [23, 27]

$$
\mathcal{L} f_{m}(z)=\left(\theta^{4}-\mu z \prod_{k=1}^{4}\left(\theta+a_{k}\right)\right) f_{m}(z)=0,
$$

where $\theta=z \frac{d}{d z}$ is the logarithmic derivative, the constants $\mu$ and $a_{k}$ are given in table 1 and we define $\tilde{\Pi}(z)=\left(f_{1}(z), f_{2}(z), f_{3}(z), f_{4}(z)\right)^{\mathrm{T}}$ as the period vector made up of four linearly independent solutions $f_{m}(z), m=1, \ldots, 4$. The tilde, $\sim$, in $\tilde{\Pi}(z)$ denotes that the period vector is not in a symplectic basis. The symplectic period vector $\Pi$ is most easily found at the $M$-point as suitable linear combinations of the solutions $f_{i}(z)$ as shown in the next section.

The Riemann symbol for the Picard-Fuchs equation (4.1) summarises the local exponents near its three singular points

$$
\mathcal{P}\left\{\begin{array}{lll}
0 & \frac{1}{\mu} & \infty \\
0 & 0 & a_{1} \\
0 & 1 & a_{2} \\
0 & 2 & a_{3} \\
0 & 1 & a_{4}
\end{array}\right\} .
$$

\footnotetext{
${ }^{12}$ We will use this notation when talking about a specific manifold. For general manifolds, we will use $X$.

${ }^{13}$ We will drop the weights in the notation in the following unless needed.
} 
From the discussion in section 3.2, we see that our models have a maximal unipotent $M$-point at $z=0$, a conifold $C$-point at $z=1 / \mu$ and at $z=\infty$ either a $F-, C$-, $K$ - or $M$-point which we will generically call the $s$-point. The complex structure moduli space is $\mathcal{M}_{c s}(X)=\mathbb{P}^{1} \backslash\{z=0, z=1 / \mu, z=\infty\}$. The periods $\Pi$ that we aim to calculate will be series solutions of the Picard-Fuchs equation expanded around different singular points and hence will have a finite radius of convergence. We label them as $\Pi_{M}, \Pi_{C}$ and $\Pi_{s}$ with radius of convergence $r_{M}, r_{C}$ and $r_{s}$ for the solutions around $M$-point, $C$-point and $s$-point respectively.

Even though we analysed all cases in detail, we will use $X_{4,2}, X_{3,3}$ and $X_{2,2,2,2}$ as the key examples having an (orbifolded) $C$-point, $K$-point and $M$-point at $\psi=0$ respectively. After this rather general discussion about the periods, let's finally calculate them.

\subsection{Symplectic basis at the $M$-point and the monodromy group $\Gamma \subset \operatorname{Sp}(4, \mathbb{Z})$}

We start with results from [23, 29], where the authors calculate the periods in a symplectic basis around the $M$-point. This point corresponds to a large radius point of the mirror family $Y$, so that the classical intersection of $Y$ that occur in the $\hat{\Gamma}$-class, cf [29], give sufficient information to construct the integral symplectic basis.

One starts with defining

$$
I_{\Gamma}(z, \epsilon)=\sum_{k=0}^{\infty} \frac{\prod_{l=1}^{n-3} \Gamma\left(d_{l}(k+\epsilon)+1\right)}{\prod_{l=1}^{n+1} \Gamma\left(w_{l}(k+\epsilon)+1\right)} z^{k+\epsilon}=\sum_{q=0}^{3} L_{q}(z)(2 \pi i \epsilon)^{q}
$$

for a given threefold $X_{d_{1}, \ldots, d_{n-3}}\left(w_{1}, \ldots, w_{n+1}\right)$. From this, $L_{q}(z)$ can be extracted by differentiating the equation (4.3) $q$ times with respect to $\epsilon$ and then taking the limit $\epsilon \rightarrow 0$. The $L_{q}(z)$ constitutes the $\mathbb{Q}^{14}$ basis which combine to give us the period in symplectic basis as $[23,29]$

$$
\Pi_{M}=\left(\begin{array}{c}
F_{0} \\
F_{1} \\
X^{0} \\
X^{1}
\end{array}\right)=\left(\begin{array}{c}
\int_{B_{0}} \Omega \\
\int_{B_{1}} \Omega \\
\int_{A_{0}} \Omega \\
\int_{A_{1}} \Omega
\end{array}\right)=\left(\begin{array}{c}
\kappa L_{3}+\frac{c_{2} \cdot D}{12} L_{1} \\
-\kappa L_{2}+\sigma L_{1} \\
L_{0} \\
L_{1}
\end{array}\right),
$$

where $\kappa$ and $c_{2} \cdot D$ are given in table 1 and $\sigma=(\kappa \bmod 2) / 2$. These periods have a radius of convergence $r_{M}=1 / \mu$, which is nothing but the distance to the nearest singular point from $M$-point. In the following, we will extend these solutions to the rest of the moduli space. It follows immediately from the logarithmic terms that the monodromy induced by a counterclockwise loop around $z=0$ transforms the period as $\Pi \rightarrow M_{M} \Pi$ with

$$
M_{M}=\left(\begin{array}{cccc}
1 & -1 & \frac{\kappa}{6}+\frac{c_{2} \cdot D}{12} & \frac{\kappa}{2}+\sigma \\
0 & 1 & \sigma-\frac{\kappa}{2} & -\kappa \\
0 & 0 & 1 & 0 \\
0 & 0 & 1 & 1
\end{array}\right)
$$

\footnotetext{
${ }^{14}$ This means that the coefficients of the linear combination of $L_{q}(z)$, which gives us the period vector, are elements of the set $\mathbb{Q}$.
} 
The hypergeometric models have only three singular points, the above type $M$-point at $z=0$, a $C$-point, which is a normal conifold at $z=\mu^{-1}$ and at $1 / z=0$ either an $F-, C$-, $K$ - or $M$-point, all of them with an orbifold action on top. Since w.r.t. to the symplectic basis (4.4) it is always the same cycle, namely the triple logarithmic one that corresponds to the $D 6$ brane on the mirror that vanishes, we can write an universal second monodromy matrix for the conifold

$$
M_{C}=\left(\begin{array}{cccc}
1 & 0 & 0 & 0 \\
0 & 1 & 0 & 0 \\
-1 & 0 & 1 & 0 \\
0 & 0 & 0 & 1
\end{array}\right)
$$

Since $\mathcal{M}_{c s}=\mathbb{P}^{1} \backslash\{z=0, \mu, \infty\}$, we get for the third monodromy which distinguishes the $F, C, K, M$ type at $\frac{1}{z}=0$

$$
M_{1 / z=0}=M_{M} M_{C}=\left(\begin{array}{cccc}
1-\frac{\kappa}{6}-\frac{c_{2} \cdot D}{12} & -1 & \frac{\kappa}{6}+\frac{c_{2} \cdot D}{12} & \frac{\kappa}{2}+\sigma \\
\frac{\kappa}{2}-\sigma & 1 & -\frac{\kappa}{2}+\sigma & -\kappa \\
-1 & 0 & 1 & 0 \\
-1 & 0 & 1 & 1
\end{array}\right)
$$

Any two of these three matrices generate the monodromy group $\Gamma \subset \operatorname{Sp}(4, \mathbb{Z})$.

\subsection{Period degeneration near the $C$-point}

To find periods around the point $\delta=1-\mu z$, we have to first solve the Picard-Fuchs equation and then find the matrix of transition to get periods in symplectic basis.

After change of variable from $z$ to $\delta$, equation (4.1) becomes

$$
\mathcal{L} f_{m}(\delta)=\left(x^{4}-(1-\delta) \prod_{k=1}^{4}\left(x+a_{k}\right)\right) f_{m}(\delta)=0,
$$

where $x=(\delta-1) \frac{d}{d \delta}$. Using a series ansatz for $f_{m}(\delta)$, we find the following basis:

- $X_{4,2}$ :

$$
\tilde{\Pi}_{C}(\delta)=\left(\begin{array}{c}
f_{1}(\delta) \\
f_{2}(\delta) \\
f_{3}(\delta) \\
f_{4}(\delta)
\end{array}\right)=\left(\begin{array}{c}
1+\frac{\delta^{3}}{256}+\frac{311 \delta^{4}}{49152}+\mathcal{O}\left(\delta^{5}\right) \\
\nu \\
\delta^{2}+\frac{119 \delta^{3}}{96}+\frac{23825 \delta^{4}}{18432}+\mathcal{O}\left(\delta^{5}\right) \\
\nu \log (\delta)-\frac{1045 \delta^{3}}{18432}-\frac{25013 \delta^{4}}{262144}+\mathcal{O}\left(\delta^{5}\right)
\end{array}\right),
$$

where $\nu=\delta+\frac{23 \delta^{2}}{32}+\frac{1745 \delta^{3}}{3072}+\frac{31087 \delta^{4}}{65536}+\mathcal{O}\left(\delta^{5}\right)$ which is the unique vanishing period.

- $X_{3,3}$ :

$$
\tilde{\Pi}_{C}(\delta)=\left(\begin{array}{c}
f_{1}(\delta) \\
f_{2}(\delta) \\
f_{3}(\delta) \\
f_{4}(\delta)
\end{array}\right)=\left(\begin{array}{c}
1+\frac{\delta^{3}}{243}+\frac{175 \delta^{4}}{26244}+\mathcal{O}\left(\delta^{5}\right) \\
\nu \\
\delta^{2}+\frac{67 \delta^{3}}{54}+\frac{7549 \delta^{4}}{5832}+\mathcal{O}\left(\delta^{5}\right) \\
\nu \log (\delta)-\frac{323 \delta^{3}}{5832}-\frac{58819 \delta^{4}}{629856}+\mathcal{O}\left(\delta^{5}\right)
\end{array}\right)
$$

where $\nu=\delta+\frac{13 \delta^{2}}{18}+\frac{139 \delta^{3}}{243}+\frac{12553 \delta^{4}}{26244}+\mathcal{O}\left(\delta^{5}\right)$ is the unique vanishing period. 
- $X_{2,2,2,2}$ :

$$
\tilde{\Pi}_{C}(\delta)=\left(\begin{array}{c}
f_{1}(\delta) \\
f_{2}(\delta) \\
f_{3}(\delta) \\
f_{4}(\delta)
\end{array}\right)=\left(\begin{array}{c}
1+\frac{\delta^{3}}{192}+\frac{13 \delta^{4}}{1536}+\mathcal{O}\left(\delta^{5}\right) \\
\nu \\
\delta^{2}+\frac{5 \delta^{3}}{4}+\frac{377 \delta^{4}}{288}+\mathcal{O}\left(\delta^{5}\right) \\
\nu \log (\delta)-\frac{13 \delta^{3}}{288}-\frac{89 \delta^{4}}{1152}+\mathcal{O}\left(\delta^{5}\right)
\end{array}\right),
$$

where $\nu=\delta+\frac{3 \delta^{2}}{4}+\frac{29 \delta^{3}}{48}+\frac{49 \delta^{4}}{96}+\mathcal{O}\left(\delta^{5}\right)$ is the unique vanishing period.

The radius of convergence of the periods calculated above is $r_{C}=1 / \mu,{ }^{15}$ which is again the distance to the nearest singular point from the $C$-point. The periods $\tilde{\Pi}_{C}$ calculated above are in an arbitrary basis, but we want periods in symplectic basis. Hence, we want to find the continuation matrix $T_{M C}$ satisfying

$$
\Pi_{C}(\delta)=T_{M C} \tilde{\Pi}_{C}(\delta) .
$$

In figure 1, we show the convergence region of the periods we have calculated. Note that $\Pi_{M}$ and $\Pi_{C}$ are both convergent in the region common to blue and red. Hence, select the point in the middle, $z=0.5 / \mu$ (or $\delta=0.5$ ), and at this point $\Pi_{M}=\Pi_{C}$ therefore we have

$$
\Pi_{C}(\delta=0.5)=T_{M C} \tilde{\Pi}_{C}(\delta=0.5)=\Pi_{M}(z=0.5 / \mu) .
$$

In the equation above, we know $\tilde{\Pi}_{C}$ and $\Pi_{M}$, and hence, we can solve for $T_{M C}$ by expanding the solutions around $z=1 / \mu$ and comparing the coefficients. Following are the $T_{M C}$ we find:

- $X_{4,2}$ :

$$
T_{M C}=\left(\begin{array}{cccc}
0 & -0.45016 i & 0 & 0 \\
7.2218 & 1.4000 & -0.20681 & 0 \\
1.0873 & -0.028048 & 0.006033 & 0.071645 \\
1.1186 i & +0.14909 i & -0.029801 i & 0
\end{array}\right)
$$

- $X_{3,3}$ :

$$
T_{M C}=\left(\begin{array}{cccc}
0 & -0.47746 i & 0 & 0 \\
7.2268+0.53267 i & 1.4974+0.074299 i & -0.22102-0.015039 i & 0 \\
1.0922 & -0.029110 & 0.006133 & 0.075991 \\
1.0653 i & 0.14859 i & -0.030072 i & 0
\end{array}\right)
$$

- $X_{2,2,2,2}$ :

$$
T_{M C}=\left(\begin{array}{cccc}
0 & -0.6366 i & 0 & 0 \\
8.9491 & 2.2373 & -0.3622 & 0 \\
1.1186 & -0.032354 & 0.0053906 & 0.10132 \\
0.90273 i & 0.14610 i & -0.032929 i & 0
\end{array}\right)
$$

\footnotetext{
${ }^{15}$ This is the convergence radius in $z$ coordinates. In $\delta$ coordinates, $r_{C}=1$.
} 


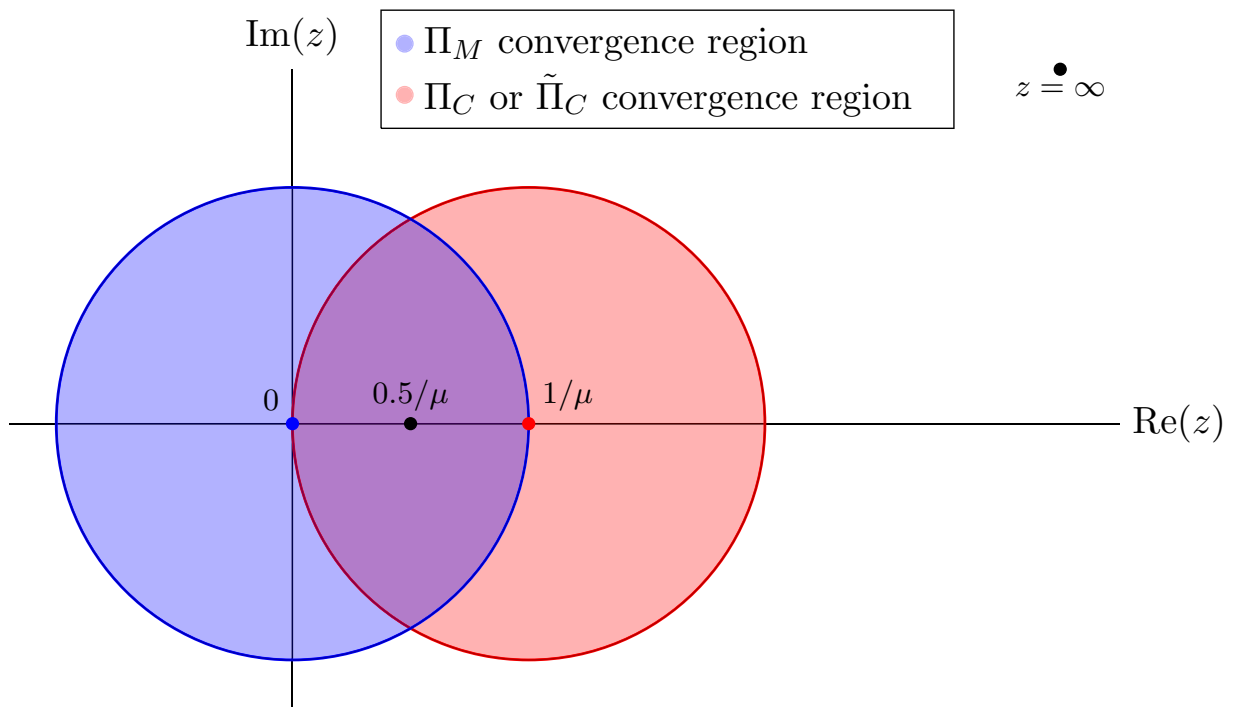

Figure 1. The convergence region of periods expanded around $M$ - and $C$ - point in $\mathcal{M}_{c s}$.

\subsection{Around $s$-Point $(\psi=0)$}

Finally, we will find the converging periods around the $s$-point at $w=1 / z=0 .{ }^{16}$ First, just like in section 4.2, we rewrite equation (4.1) after change of variables from $z$ to $w$, getting

$$
\mathcal{L} f_{m}(w)=\left(\vartheta^{4}-\mu \frac{1}{w} \prod_{k=1}^{4}\left(\vartheta-a_{k}\right)\right) f_{m}(w)=0,
$$

where $\vartheta=w \frac{d}{d w}$. This equation can be solved using Meijer G-functions and the complete calculation is shown in appendix A. Here, we will just state the resulting functions.

- $X_{4,2}$ :

$$
\begin{aligned}
& f_{1}(w)=G_{4,4}^{1,4}\left(-\frac{w}{2^{10}} \mid \begin{array}{c}
1,1,1,1 \\
\frac{1}{4}, \frac{1}{2}, \frac{1}{2}, \frac{3}{4}
\end{array}\right), \quad f_{2}(w)=G_{4,4}^{1,4}\left(-\left.\frac{w}{2^{10}}\right|_{\frac{1}{2}, \frac{1}{4}, \frac{1}{2}, \frac{3}{4}} ^{1,1,1,1}\right), \\
& f_{3}(w)=G_{4,4}^{1,4}\left(-\frac{w}{2^{10}} \mid \begin{array}{l}
1,1,1,1 \\
\frac{3}{4}, \frac{1}{4}, \frac{1}{2}, \frac{1}{2}
\end{array}\right) \text { and } \quad f_{4}(w)=G_{4,4}^{2,4}\left(\left.\frac{w}{2^{10}}\right|_{\frac{1}{2}, \frac{1}{2}, \frac{1}{4}, \frac{3}{4}} ^{1,1,1,1}\right) \text {. }
\end{aligned}
$$

- $X_{3,3}$ :

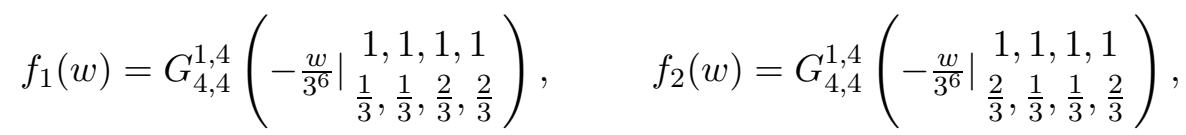

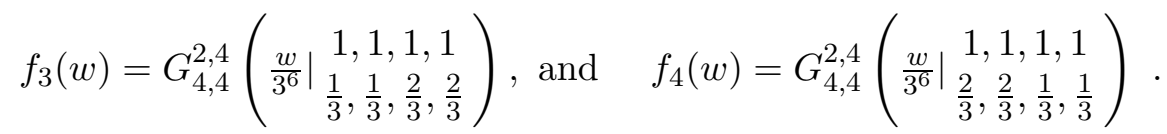

\footnotetext{
${ }^{16}$ To increase confusion, we have three variables now: $z, w$ and $\psi$ and we will use them interchangeably whenever convenient.
} 
- $X_{2,2,2,2}$ :

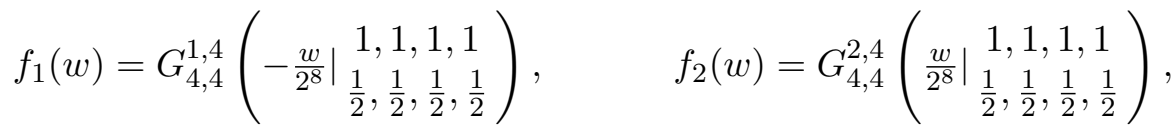

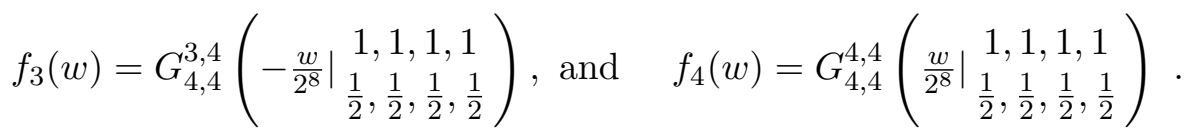

These functions have a Mellin-Barnes type integral representation which allows us to find converging solutions in the region $|w|<\mu$ and $|w|>\mu$ by changing the contour (see appendix A). This provides us with an analytic continuation of the period $\tilde{\Pi}_{s}(w)=$ $\left(f_{1}(w), f_{2}(w), f_{3}(w), f_{4}(w)\right)^{\mathrm{T}}$ from $|w|<\mu$ to $|w|>\mu$. Using the form of $\tilde{\Pi}_{s}(w)$ in $|w|>\mu$ (or $|z|<1 / \mu$ ), and matching first few coefficients with $\Pi_{M}(z)$, we can find the transition matrix $T_{M s}$ satisfying

$$
\Pi_{M}(z)=T_{M s} \tilde{\Pi}_{s}(w)
$$

The resulting matrices are:

- $X_{4,2}$ :

$$
T_{M s}=\left(\begin{array}{cccc}
\frac{1+i}{2 \pi^{2}} & 0 & -\frac{1-i}{2 \pi^{2}} & -\frac{2 i \sqrt{2}}{\pi^{3}} \\
\frac{1-i}{\pi^{2}} & \frac{2 i \sqrt{2}}{\pi^{2}} & -\frac{1+i}{\pi^{2}} & 0 \\
\frac{1}{2 \pi^{2}} & \frac{i \sqrt{2}}{\pi^{2}} & -\frac{1}{2 \pi^{2}} & -\frac{i \sqrt{2}}{\pi^{3}} \\
\frac{1+i}{4 \pi^{2}} & 0 & -\frac{1-i}{4 \pi^{2}} & -\frac{i}{\sqrt{2} \pi^{3}}
\end{array}\right),
$$

- $X_{3,3}$ :

$$
T_{M s}=\left(\begin{array}{cccc}
-\frac{9(3+i \sqrt{3})}{8 \pi^{2}} & \frac{9(3-i \sqrt{3})}{8 \pi^{2}} & \frac{9 i}{8 \pi^{3}} & \frac{9 i}{8 \pi^{3}} \\
-\frac{3+i \sqrt{3}}{8 \pi^{2}} & \frac{3-i \sqrt{3}}{8 \pi^{2}} & \frac{21(1-i \sqrt{3})}{8(\sqrt{3}-5 i) \pi^{3}} & \frac{3 i(4 \sqrt{3}+i)}{4(\sqrt{3}-5 i) \pi^{3}} \\
-\frac{3(5+i \sqrt{3})}{8 \pi^{2}} & \frac{3(5-i \sqrt{3})}{8 \pi^{2}} & \frac{3(\sqrt{3}+3 i)}{16 \pi^{3}} & -\frac{3(\sqrt{3}-3 i)}{16 \pi^{3}} \\
-\frac{3+i \sqrt{3}}{4 \pi^{2}} & \frac{3-i \sqrt{3}}{4 \pi^{2}} & \frac{3 i}{8 \pi^{3}} & \frac{3 i}{8 \pi^{3}}
\end{array}\right),
$$

- $X_{2,2,2,2}$ :

$$
T_{M s}=\left(\begin{array}{cccc}
0 & -\frac{4 i}{\pi^{3}} & 0 & \frac{2 i}{\pi^{5}} \\
0 & \frac{4 i}{\pi^{3}} & -\frac{4 i}{\pi^{4}} & 0 \\
\frac{i}{\pi^{2}} & -\frac{i}{\pi^{3}} & -\frac{i}{\pi^{4}} & \frac{i}{\pi^{5}} \\
0 & -\frac{i}{2 \pi^{3}} & 0 & \frac{i}{2 \pi^{5}}
\end{array}\right)
$$

Using the $T_{M s}$, we can find periods in symplectic basis for $|z|>1 / \mu$ using

$$
\Pi_{s}(w)=T_{M s} \tilde{\Pi}_{s}(w) .
$$




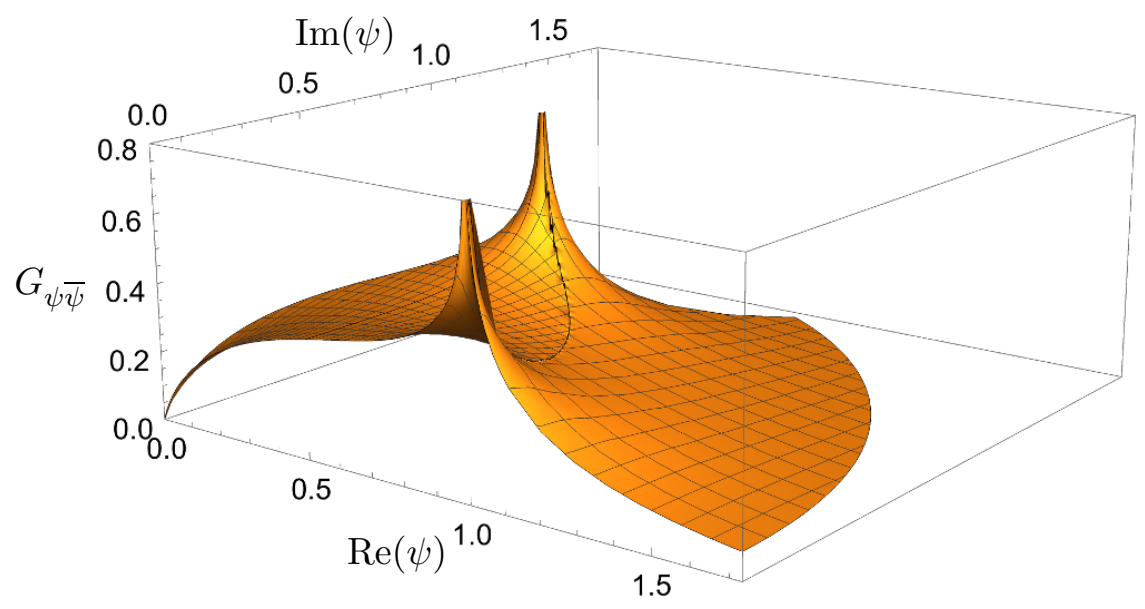

Figure 2. The metric $G_{\psi \bar{\psi}}$ plotted as function of $\psi$ for $\mathcal{M}_{c s}\left(X_{4,2}\right)$.

\begin{tabular}{|c|c|c|}
\hline$\psi$ & $G_{\psi \bar{\psi}}$ & $R$ \\
\hline 0 & $-\frac{27 \pi^{2} \Gamma\left(-\frac{1}{4}\right)^{4}|\psi| \log (|\psi|)}{8 \Gamma\left(\frac{1}{4}\right)^{8}}$ & $-\frac{4 \Gamma\left(\frac{1}{4}\right)^{8}}{\left.27 \pi^{2} \Gamma\left(-\frac{1}{4}\right)^{4}|\psi|^{3} \log ^{3}(|\psi|)\right)}$ \\
\hline 1 & $-\frac{6^{2} \kappa \log (r)}{(2 \pi)^{3} \mathrm{ag}}$ & $-\frac{\mathrm{ag}(2 \pi)^{3}}{2 \times 6^{2} \kappa r^{2} \log ^{3}(r)}$ \\
\hline$\infty$ & $\frac{3}{4|\psi|^{2} \log ^{2}(|\psi|)}$ & $-\frac{4}{3}$ \\
\hline
\end{tabular}

Table 2. The behaviour of $G_{\psi \bar{\psi}}$ as $\psi$ approaches the singular points for $\mathcal{M}_{c s}\left(X_{4,2}\right)$. Here $r=$ $|\psi-1|$, a is real part of $\left(T_{M C}\right)_{2,1}, \mathrm{~g}$ is imaginary part of $\left(T_{M C}\right)_{4,1}$, the matrix $T_{M C}$ is given in equation (4.14) and $\kappa=8$ is given in table 1.

\section{Global structure of the metric on $\mathcal{M}_{c s}(X)$}

With the periods calculated on the whole moduli space $\mathcal{M}_{c s}$, we can now find the Kähler potential using (2.8) and then find the metric and its scalar curvature using equation (2.2) and equation (2.3) respectively. In the following, we will plot the resulting metric for each threefold's complex structure moduli space and talk qualitatively about the distances to the singular points. These distances are measured from any non-singular point on the threefold.

- $X_{4,2}$ : the metric for this threefold is shown in figure 2 , and table 2 shows the limiting behaviour of the metric and the scalar curvature as we approach the singular points. From the limiting behaviour, we can see that there is only one point at infinite distance, the $M$-point at $\psi=\infty$. The point $\psi=0$ is at finite distance. At the conifold point, $\psi=1$, the metric and curvature diverges but the distance is finite.

- $X_{3,3}$ : for this case, figure 3 shows the metric and table 3 shows limiting behaviour of the metric and the scalar curvature. Similar to $X_{4,2}$ discussed above, for this threefold $M$-point is at infinite distance and the conifold point is finitely away from any non-singular point. Here, a new feature is observed at the $s$-point, it is at infinite distance. 


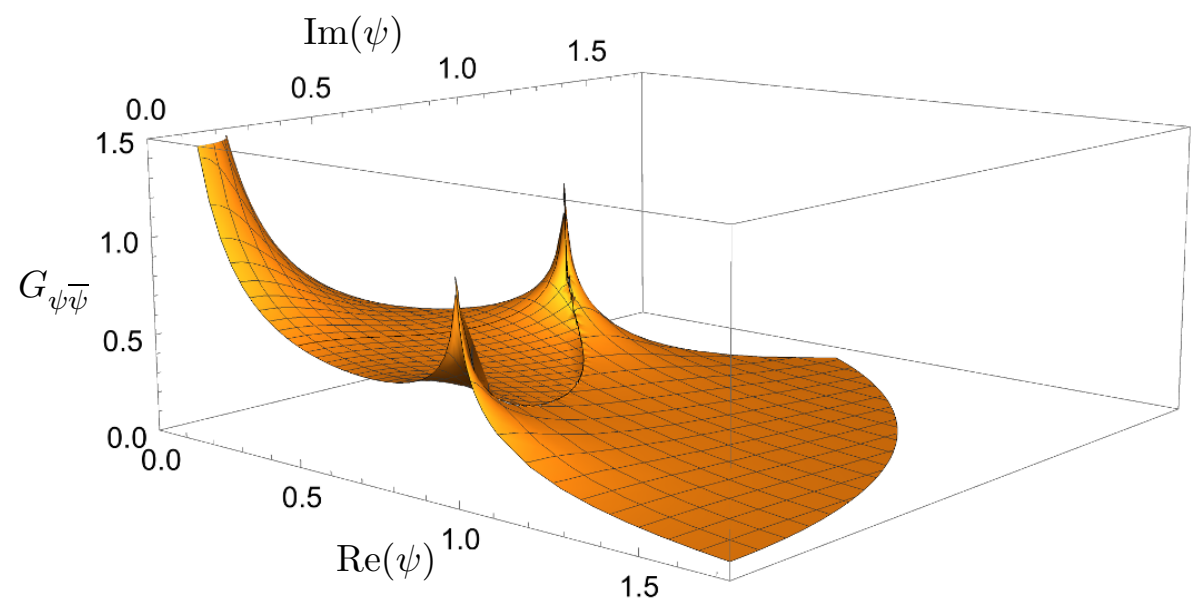

Figure 3. The metric $G_{\psi \bar{\psi}}$ plotted as function of $\psi$ for $\mathcal{M}_{c s}\left(X_{3,3}\right)$.

\begin{tabular}{|c|c|c|}
\hline$\psi$ & $G_{\psi \bar{\psi}}$ & $R$ \\
\hline 0 & $\frac{1}{4|\psi|^{2} \log ^{2}(|\psi|)}$ & -4 \\
\hline 1 & $-\frac{6^{2} \kappa \log (r)}{(2 \pi)^{3} \mathrm{ag}}$ & $-\frac{\mathrm{ag}(2 \pi)^{3}}{2 \times 6^{2} \kappa r^{2} \log ^{3}(r)}$ \\
\hline$\infty$ & $\frac{3}{4|\psi|^{2} \log ^{2}(|\psi|)}$ & $-\frac{4}{3}$ \\
\hline
\end{tabular}

Table 3. The behaviour of $G_{\psi \bar{\psi}}$ as $\psi$ approaches the singular points for $\mathcal{M}_{c s}\left(X_{3,3}\right)$. Here $r=$ $|\psi-1|$, a is real part of $\left(T_{M C}\right)_{2,1}, \mathrm{~g}$ is imaginary part of $\left(T_{M C}\right)_{4,1}$, the matrix $T_{M C}$ is given in equation (4.15) and $\kappa=9$ is given in table 1.

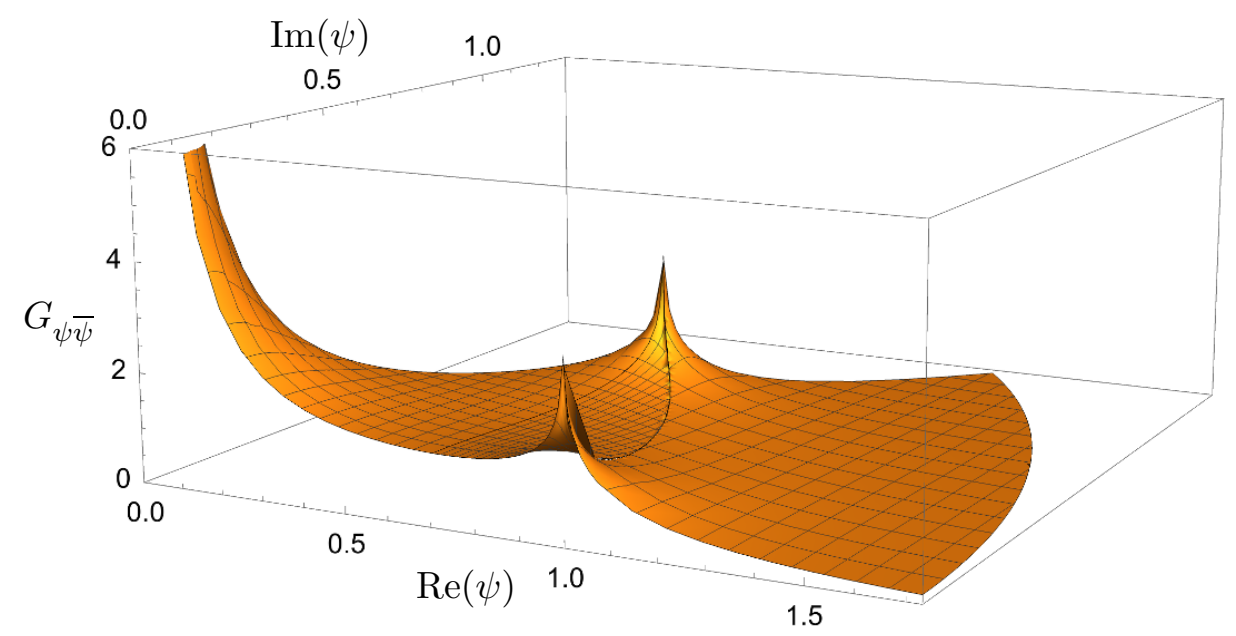

Figure 4. The metric $G_{\psi \bar{\psi}}$ plotted as function of $\psi$ for $\mathcal{M}_{c s}\left(X_{2,2,2,2}\right)$.

- $X_{2,2,2,2}$ : for this case, figure 4 shows the metric and table 4 has information about limiting behaviour of the metric and the scalar curvature. The metric for this threefold is qualitatively similar to that of $X_{3,3}$ with a more severe singularity at $\psi=0$. 


\begin{tabular}{|c|c|c|}
\hline$\psi$ & $G_{\psi \bar{\psi}}$ & $R$ \\
\hline 0 & $\frac{3}{4|\psi|^{2} \log ^{2}(|\psi|)}$ & $-\frac{4}{3}$ \\
\hline 1 & $-\frac{8^{2} \kappa \log (r)}{(2 \pi)^{3} \mathrm{ag}}$ & $-\frac{\mathrm{ag}(2 \pi)^{3}}{2 \times 8^{2} \kappa r^{2} \log ^{3}(r)}$ \\
\hline$\infty$ & $\frac{3}{4|\psi|^{2} \log ^{2}(|\psi|)}$ & $-\frac{4}{3}$ \\
\hline
\end{tabular}

Table 4. The behaviour of $G_{\psi \bar{\psi}}$ as $\psi$ approaches the singular points for $\mathcal{M}_{c s}\left(X_{2,2,2,2}\right)$. Here $r=|\psi-1|$, a is real part of $\left(T_{M C}\right)_{2,1}, \mathrm{~g}$ is imaginary part of $\left(T_{M C}\right)_{4,1}$, the matrix $T_{M C}$ is given in equation (4.16) and $\kappa=16$ is given in table 1.

To summarise, we have two points of special interest: $\psi=\infty$ which is at infinite distance for every threefold, and $\psi=0$ which is at infinite distance for $X_{3,3}$ and $X_{2,2,2,2}$. If SDC holds, we expect to find a tower of exponentially light states as we approach these points. We will discuss each of the two points in the following.

\section{Swampland distance conjecture}

In the following, we will look at the two infinite distance points discussed above. We will identify the light states with $D 2-D 0$ bound states at the $M$-point and since we don't have a type IIA interpretation yet, at the $K$-point, we will identify the light states with BPS 3-branes.

\subsection{Light states near the $M$-point}

Light BPS states can be identified by their contribution to the Schwinger one loop amplitude creating the coupling of the anti selfdual part of the curvature $R_{-}$to the anti selfdual part of the graviphoton field strength $T_{-}$. This term resides in the vector multiplet sector of the effective $4 \mathrm{~d} N=2$ action and its dependence on the Kähler moduli $t$ is given by the higher genus topological string amplitudes $\mathcal{F}_{g}(t)$ as

$$
\sum_{g>0} \int_{M_{1,3}} d x^{4} \mathcal{F}_{g}(t) T_{-}^{2 g-2} R_{-}^{2}
$$

As pointed out in $[30,31]$, this moduli dependence can be calculated by the Schwinger oneloop integral shown in figure 5, where the topological string coupling $\lambda$ becomes formally identified with the $T_{-}$insertions.

Generally the BPS states contribute to the Schwinger one loop amplitude only via a BPS index. In the large volume limit the index $I_{g}^{\beta}$ is directly related to the BPS degeneracies $N_{j_{L} j_{R}}^{\beta}$ by the following re-organisation of the spin representations $\left[j_{L}\right]_{L},\left[j_{R}\right]_{L}$ of the 5 dittle group $\mathrm{SU}(2)_{L} \times \mathrm{SU}(2)_{R}$

$$
\sum_{J_{L} j_{R} \in \frac{1}{2} \mathbb{N}}(-1)^{j_{R}}\left(2 J_{+}+1\right) N_{j_{L} j_{R}}^{\beta}\left[j_{L}\right]_{L}=\sum_{g=0}^{\infty} I_{g}^{\beta}\left(2[0]_{L}+\left[\frac{1}{2}\right]\right)^{\otimes g} .
$$




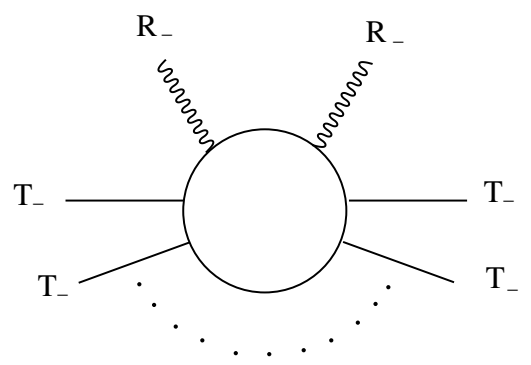

Figure 5. One loop integral counting the BPS index $I_{g}^{\beta}$ with BPS states running in the loop.

As explained in [31, 32] and later (mathematically) rigorously in [33] using stable pair invariants, these BPS invariants can be viewed in the large volume limit as bound states of $D 2$ branes interpreted as pure sheaf $\mathcal{F}$ of complex dimension one carrying the charge $\operatorname{ch}_{2}(\mathcal{F})=\beta \in H_{2}(M, \mathbb{Z})$ and $D 0$ branes whose charge is related to $n=\chi(\mathcal{F}) \in \mathbb{Z}$ and becomes after some one-to-one mapping identified with the genus of the topological string amplitude.

The result of the combined analysis in $[30,31]$ is that the all genus free energy of the topological string can be written as

$$
\begin{aligned}
\mathcal{F}(\lambda, t) & =\sum_{g=0}^{\infty} \lambda^{2 g-2} \mathcal{F}_{g}(t) \\
& =\frac{c(t)}{\lambda^{2}}+l(t)+\sum_{g=0}^{\infty} \sum_{\beta \in H_{2}(M, \mathbb{Z})} \sum_{m=1}^{\infty} I_{g}^{\beta} \frac{1}{m}\left(2 \sin \frac{m \lambda}{2}\right)^{2 g-2} Q^{\beta m},
\end{aligned}
$$

with $Q^{\beta}=e^{2 \pi i t_{\beta}}$, with $t_{\beta}$ unique Kähler parameters. Note that here $\beta$ is an index and for one-parameter cases we will just have $t$. Here $\mathcal{F}_{0}$, the genus zero contribution, is the prepotential, calculated using (4.4). However the classical terms, i.e. the inhomogeneous cubic polynomial $c(t)$ in $\mathcal{F}_{0}$ and the linear term $l(t)$ in $\mathcal{F}_{1}$ in $t \sim \log (z)$ are not calculated by the Schwinger loop contribution in [31]. ${ }^{17}$

Linear logarithmic contributions to $\mathcal{F}_{0}$ have been calculated from the one loop wave function regularisation contributing to the gauge coupling of one photon ${ }^{18}$ in the generic $\mathrm{U}(1)^{h_{11}}$ gauge group in the vector moduli space of Calabi-Yau compactifications, i.e. in $N=2$ supergravity, in [34] at the conifold point analogously as in $N=2$ gauge theory in [9] at the monopole point. In both cases, there is only one particle namely the magnetic monopole or the massless extremal black hole running the loop and the calculation is simple.

In the context of the SDC it would be satisfying to show that the integrating out the infinite tower of states yields the structure of the higher logarithmic terms in $\mathcal{F}_{0}$ at the $M$-point (and the $K$ - points) that give rise to the infinite metric distance property. While we have not attempted this calculation in this project, ${ }^{19}$ it is likely that it will work only

\footnotetext{
${ }^{17}$ Except for the constant term $\frac{\chi \zeta(3)}{2(2 \pi i)^{3}}$ in $c(t)$, which comes from pure $D 0$ brane charges and gives only a sub-leading contribution to the metric.

${ }^{18}$ This photon is the one that couples to the light charges state.

${ }^{19} \mathrm{~A}$ heuristic calculation using a massless scalar field and a tower of heavy states becoming light has been done, in this context, in [11].
} 
with the particular spectrum encoded in (6.3) as the asymptotic growth of these states knows about the classical intersection numbers that are the coefficients of the cubic in $c(t)$ as it is predicted from the microscopic black hole entropy counting arguments and was checked in [35].

While the result (6.3) is specific for the $D 2-D 0$ brane spectrum at large radius points, the evaluation of the Schwinger one-loop diagram is possible whenever the BPS spectrum is known at a singular point and also the topological string amplitudes can be calculated at other singular points in the B-model [10]. For example the contribution of the light particle at the conifold to the $\mathcal{F}_{g>0}\left(t_{c}\right)$ amplitudes in the leading order in the flat coordinate $t_{c}$ at the conifold has been evaluated via the Schwinger loop calculation and gives rise to the conifold gap [10]. The logarithmic contribution to $\mathcal{F}_{1}\left(t_{c}\right)=\frac{1}{12} t_{c}+\ldots$ at the conifold has been obtained previously in [36]. Also, one can perform the calculation to find the behaviour of a state which becomes massless at $C$-point. For the exponents $(a, b, b, c)$, near the singularity, one finds

$$
m \sim \alpha|\tilde{x}|^{b-a}, \quad d\left(\tilde{x}, r_{0}\right)=-\beta \int_{r_{0}}^{|\tilde{x}|} d x\left(-x^{-2 a} \log (x)\right)^{1 / 2},
$$

where $\tilde{x}$ is the local coordinate such that singular point is at $\tilde{x}=0, r_{0}>\tilde{x}, d\left(\tilde{x}, r_{0}\right)$ is the distance between $\tilde{x}$ and $r_{0}$, and $\alpha, \beta \in \mathbb{R}^{+}$. Clearly, mass vanishes at $\tilde{x}=0$, and distance to singularity, i.e. $d\left(0, r_{0}\right)$, is finite since $a<1 / 2$. One can find the coordinate $\tilde{x}$ in terms of distance $d\left(\tilde{x}, r_{0}\right)$ by inverting the second function above.

In any case at the $M$ - points the $D 2-D 0$ bound states in the type IIA compactification are the desired infinite tower of stable states, whose mass of the constituents vanishes exponentially with the distance as one approaches the $M$-point,

$$
m_{0}^{p} \sim e^{-\sqrt{3} d(p, q)} m_{0}^{q}, \quad m_{2}^{p} \sim e^{-\frac{1}{\sqrt{3}} d(p, q)} m_{2}^{q}
$$

and is dominated by the $D 2$-brane mass. This result is calculated by performing analogous analysis to that presented in detail in section 6.2. Moreover in the topological sector, mirror symmetry applies and yields the very same prediction for the type IIB light BPS states.

Let us compare this behaviour with the one of Kaluza Klein states proposed in [12]. On performing dimensional reduction, one finds that the mass of these KK states scales as

$$
m_{K K}^{2} \sim \frac{1}{R^{2}}
$$

where $R^{2}$ is the size of the manifold. For the threefolds considered here, in the large volume limit, $R^{2}$ is given by the imaginary part of the Kähler modulus (area), $t$, on $\mathcal{M}_{c k s}(Y)$ [37]. The mirror map is given by [38]

$$
t(z)=\frac{X^{1}(z)}{X^{0}(z)}=B+i J
$$

where $X^{1}(z)$ and $X^{0}(z)$ are elements of the period vector (see equation (2.6)), $B=\int_{\mathcal{C}} b$ is the Neveu-Schwarz $B$-field flux around the 2-cycle $\mathcal{C}$ forming a basis of $H_{2}(Y)$ and $J=\int_{\mathcal{C}} \omega$ is the size of the 2-cycle. 
Near $\psi=\infty$ (or $z=0$ ), we can use equation (4.4) and (4.3) to write

$$
\begin{aligned}
t(z) & =\frac{1}{2 \pi i} \frac{\lim _{\epsilon \rightarrow 0} \frac{\partial I_{\Gamma}(z, \epsilon)}{\partial \epsilon}}{I_{\Gamma}(z, 0)} \\
& =\frac{1}{2 \pi i} \log (z)+h(z),
\end{aligned}
$$

where

$$
\begin{aligned}
h(z) & =\frac{1}{2 \pi i} \frac{\sum_{k=0}^{\infty}\left(\lim _{\epsilon \rightarrow 0} \frac{\partial g(k, \epsilon)}{\partial \epsilon}\right) z^{k}}{\sum_{k=0}^{\infty} g(k, 0) z^{k}}, \text { with } \\
g(k, \epsilon) & =\frac{\Gamma\left(d_{1}(k+\epsilon)+1\right) \ldots \Gamma\left(d_{n-3}(k+\epsilon)+1\right)}{\Gamma(k+\epsilon+1)^{n+1}} .
\end{aligned}
$$

In the limit $z \rightarrow 0$, we get

$$
h(z) \rightarrow \frac{1}{2 \pi i} \frac{\gamma\left(n+1-\sum_{i=1}^{n-3} d_{i}\right)+\mathcal{O}(z)}{1+\mathcal{O}(z)} \stackrel{(3.21)}{=} \mathcal{O}(z),
$$

where $\gamma \sim 0.5772$ is the Euler-Mascheroni constant.

Hence, the mass, $m_{K K}$, mentioned in equation (6.6), in the limit $z \rightarrow 0$, can be calculated using equation (6.8) and equation (6.10) as

$$
\begin{aligned}
m_{K K}^{2} \sim \frac{1}{R^{2}} \sim \frac{1}{\Im(t(z))} & =-\frac{2 \pi}{\log (|z|)}+\mathcal{O}\left(\frac{|z|}{\log (|z|)}\right) \\
\stackrel{(3.22)}{=} & \frac{2 \pi}{\log \left(\mu|\psi|^{n+1}\right)}+\mathcal{O}\left(\frac{1}{|\psi|^{n+1} \log (|\psi|)}\right) \\
& =\frac{2 \pi}{n+1} \frac{1}{\log (|\psi|)}+\mathcal{O}\left(\frac{1}{\log ^{2}(|\psi|)}\right) .
\end{aligned}
$$

Next, consider a point $\psi_{0} \gg 1$ on the moduli space. For another point $\psi_{l}>\psi_{0}$, we can find the distance between $\psi_{l}$ and $\psi_{0}$ using the metric in table 2,3 or 4

$$
d\left(\psi_{l}, \psi_{0}\right) \sim \int_{\psi_{0}}^{\psi_{l}} \sqrt{\frac{3}{4|\psi|^{2} \log ^{2}(|\psi|)}} d|\psi|=\frac{\sqrt{3}}{2} \log \left(\frac{\log \left(\left|\psi_{l}\right|\right)}{\log \left(\left|\psi_{0}\right|\right)}\right) .
$$

Finally, combining equation (6.12) and (6.11), we can find the relation between the mass of the KK states and the distance on the moduli space,

$$
m_{K K}^{l} \sim \frac{1}{\sqrt{\log \left(\left|\psi_{l}\right|\right)}} \sim m_{K K}^{0} e^{-\frac{1}{\sqrt{3}} d\left(\psi_{l}, \psi_{0}\right)} .
$$

Of course since the argument of [12] is based on dimensional- and scale arguments relating in particular the KK scale to the area as measured by the Kähler parameter it gives the same exponentially behaviour as the one for the $D 2-D 0$ brane BPS states, without making statements about their detailed multiplicity. In [12], the authors also construct geodesics on the moduli space to verify the RSDC. We will not reproduce the corresponding calculation for all cases, but qualitatively, one can see that the RSDC is true for generic $M$-points. Next, we come to the conceptually more challenging cases that occur near the $\psi=0$ point, which we also call the $s$-point. 


\subsection{Light states near the $s$-point}

In this section we try to construct the tower of light states to support the SDC at s-point which is at infinite distance for $X_{3,3}$ and $X_{2,2,2,2}$. The candidates are the 3-branes in type IIB theory which come from reducing the RR 4 -form potential $C^{(4)}$ on the $A^{I}$ cycles. On the $B_{I}$ cycles, we get their electromagnetic duals. The periods can be used to calculate their central charge, at a point on the moduli space, using

$$
Z_{\mathbf{q}}=e^{K / 2}\left(\mathbf{q}^{\mathrm{T}} \Sigma \Pi\right)
$$

where $K$ is the Kähler potential (see equation (2.8)), $\mathbf{q}$ is the (electric + magnetic) charge vector, $\Sigma$ is given in equation (2.9) and $\Pi$ is the period vector.

There is no reason that the 3-branes described above with information of $\mathbf{q}$ are actual, physical BPS states. This is essentially an assumption here. However, what we are able to do here, is checking if these branes remain stable as one approaches the singular point. For that, let's first write down the mass of a physical state. The mass of a BPS 3-brane state is given by [39]

$$
m_{\mathbf{q}}=\left|Z_{\mathbf{q}}\right| \text {. }
$$

The charges $\mathbf{q}$ form a charge lattice and in general, on approaching $\psi=0$, a state will not become massless, but a subset of the charge lattice becomes massless. In the following, we consider $X_{3,3}$ and $X_{2,2,2,2}$, and see what this subset looks like.

\subsection{1 $X_{3,3}$}

Since we are looking at the states when we approach $\psi=0$, we will use the period vector calculated in section 4.3. Let's first see the behaviour of the $e^{K / 2}$ term in the central charge given in equation (6.14). Using the period vector, we find (see equation (2.8))

$$
e^{K / 2}=-\frac{3^{3 / 4} \pi^{15 / 4}}{\Gamma\left(-\frac{2}{3}\right)^{3} \Gamma\left(\frac{1}{6}\right)^{3 / 2}|\psi|^{2} \sqrt{-\log (|\psi|)}}+\mathcal{O}\left(\frac{1}{|\psi|^{2}(-\log (|\psi|))^{3 / 2}}\right) .
$$

Next we want to look at the behaviour of the term $\mathbf{q}^{T} \Sigma \Pi$ in equation (6.14). For a general charge vector $\mathbf{q}$, the structure of periods near the $s$-point gives us a leading (largest) contribution of order $\psi^{2} \log (\psi)$ which would give a divergent mass when multiplied by $e^{K / 2}$ on approaching $\psi=0$.

Therefore, we next find the subset of the charge lattice which strips the combination $\mathbf{q}^{\mathrm{T}} \Sigma \Pi$ of logarithmic terms. This gives us

$$
\mathbf{q}=\left(\begin{array}{c}
x \\
y \\
\frac{4 x}{9}+\frac{y}{3} \\
\frac{x}{3}
\end{array}\right),
$$

where $x$ and $y$ are arbitrary integers such that $x \bmod 3=0$ and $(4 x+3 y) \bmod 9=0$ (quantisation condition). This subset of charge lattice gives us the light states we are looking for and we denote it by

$$
\mathcal{Q}=\{\mathbf{q} \mid x, y \in \mathbb{Z}, x \bmod 3=0,(4 x+3 y) \bmod 9=0\} \backslash\left\{(0,0,0,0)^{\mathrm{T}}\right\} .
$$


For the elements in this sub lattice, we find

$$
Z_{\mathbf{q}}=e^{K / 2}\left(\frac{\sqrt[6]{-1} \sqrt[3]{2} \Gamma\left(-\frac{2}{3}\right)^{4} \Gamma\left(\frac{1}{6}\right)(-14 \sqrt{3} x+3(\sqrt{3}+9 i) y)}{81(5 \sqrt{3}+3 i) \pi^{7 / 2}} \psi^{2}+\mathcal{O}\left(\psi^{4}\right)\right) .
$$

This gives us the mass

$$
\begin{aligned}
m_{\mathbf{q}}=\left|Z_{\mathbf{q}}\right| & =e^{K / 2}\left|\mathbf{q}^{\mathrm{T}} \Sigma \Pi\right| \\
& =\frac{-\pi^{1 / 4} \Gamma\left(-\frac{2}{3}\right)|14 \sqrt{3} x-3(9 i+\sqrt{3}) y|}{27 \times 2^{2 / 3} \times 3^{3 / 4} \sqrt{7 \Gamma\left(\frac{1}{6}\right)} \sqrt{-\log (|\psi|)}}+\mathcal{O}\left(\frac{|\psi|^{2}}{\sqrt{-\log (|\psi|)}}\right) .
\end{aligned}
$$

Now consider two points $P$ and $Q$ near $\psi=0$ such that $\left|\psi_{P}\right|>\left|\psi_{Q}\right|$, then we can calculate the distance between these two points using metric in table 3 . We get

$$
d_{P Q} \sim \int_{\psi_{P}}^{\psi_{Q}} \mathrm{~d}|\psi| \sqrt{\frac{1}{4|\psi|^{2} \log ^{2}(|\psi|)}}=\frac{1}{2} \log \left(\frac{\log \left(\left|\psi_{Q}\right|\right)}{\log \left(\left|\psi_{P}\right|\right)}\right) .
$$

Combining this with the leading term of mass of the brane calculated in equation (6.20) we can find the ratio of masses at $Q$ and $P$,

$$
\frac{m_{\mathbf{q}}^{Q}}{m_{\mathbf{q}}^{P}} \sim \sqrt{\frac{\log \left(\left|\psi_{P}\right|\right)}{\log \left(\left|\psi_{Q}\right|\right)}} \sim e^{-d_{P Q}} .
$$

Therefore, we observe that for the subset of charge lattice given by $\mathcal{Q}$, the states exponentially become massless as one approaches the $s$-point. If we can show that there are infinitely many such states which are stable, it will be an evidence of the SDC.

In the following, we will construct a subset of the charge lattice $\mathcal{Q}$ which, if assumed to be physically existing close to the singular point, will be stable as one approaches the singular point.

\subsubsection{Stability of branes in the $X_{3,3}$ model}

Let's start with the observation that the central charge of a state can be decomposed as (see equation (6.17))

$$
\begin{aligned}
Z_{\mathbf{q}} & =e^{K / 2}\left(\mathbf{q}^{\mathrm{T}} \Sigma \Pi\right) \\
& =x \Phi+y \Psi
\end{aligned}
$$

where

$$
\begin{gathered}
\Phi=e^{K / 2}\left(\mathbf{q}_{1}^{\mathrm{T}} \Sigma \Pi\right) \text { with } \quad \mathbf{q}_{1}=\left(\begin{array}{c}
1 \\
0 \\
4 / 9 \\
1 / 3
\end{array}\right) \text { and } \\
\Psi=e^{K / 2}\left(\mathbf{q}_{2}^{\mathrm{T}} \Sigma \Pi\right) \text { with } \quad \mathbf{q}_{2}=\left(\begin{array}{c}
0 \\
1 \\
1 / 3 \\
0
\end{array}\right) .
\end{gathered}
$$


Note that the states with vanishing mass are linear combination of the vanishing cycles since

$$
\begin{aligned}
\frac{\Phi}{e^{K / 2}} & =\frac{7(\sqrt{3}+3 i) f_{1}(w)+(13 \sqrt{3}-9 i) f_{2}(w)}{12(\sqrt{3}-5 i) \pi^{2}}, \\
\frac{\Psi}{e^{K / 2}} & =\frac{(3+i \sqrt{3}) f_{1}(w)+i(\sqrt{3}+3 i) f_{2}(w)}{8 \pi^{2}}
\end{aligned}
$$

where $f_{1}(w)$ and $f_{2}(w)$ are given in equation (4.19).

Now, as noted in [9], consider the possible decay process of a BPS 3-brane labelled $A$ with $Z_{\mathbf{q}(A)}=x \Phi+y \Psi$ decaying to states labelled $B_{i}$ with $Z_{\mathbf{q}\left(B_{i}\right)}=x_{i} \Phi+y_{i} \Psi$. Conservation of charge requires that

$$
Z_{\mathbf{q}(A)}=\sum_{i} Z_{\mathbf{q}\left(B_{i}\right)}
$$

however, triangle inequality tells us that

$$
m_{\mathbf{q}(A)} \leq \sum_{i} m_{\mathbf{q}\left(B_{i}\right)}
$$

where $m_{\mathbf{q}}=\left|Z_{\mathbf{q}}\right|$. This inequality is saturated when all the charges $Z_{\mathbf{q}\left(B_{i}\right)}$ are aligned. Hence, for brane $A$ to decay into the branes $B_{i}$, the charges of the branes $B_{i}$ must be aligned otherwise the brane $A$ will be stable against this decay. Note that this condition is necessary but not sufficient. Also, this condition defines the walls of marginal stability.

Assuming that $\Phi$ and $\Psi$ in equation (6.23) are not aligned, i.e. $\Phi / \Psi$ is not real, the decay of $A$ into $B_{i}$ (or vice-versa) is only possible if $(x, y)$ is proportional to $\left(x_{i}, y_{i}\right)$. In other words, if $(x, y)=(n k, n l)$ for some $n \in \mathbb{Z}$ and $k, l \in \mathbb{Z}$ satisfying $k \bmod 3=0$ and $(4 k+3 l) \bmod 9=0$ (quantisation condition). On the contrary, if $x$ and $y$ are relative primes, the state will be stable against such a decay. Since there are infinitely many relative primes in the set $\mathcal{Q}$ given in equation (6.18), we have infinitely many light stable states if $\Phi / \Psi \notin \mathbb{R}$.

With the knowledge of periods on the whole moduli space, we can indeed compute $\Phi$ and $\Psi$. More importantly, we look at the difference in the argument of $\Phi$ and $\Psi$ or $\arg (\Phi / \Psi)$. The plot is given in figure 6. In the figure, we plot $\arg (\Phi / \Psi)$ against $|\psi|$ for $\arg (\psi)=2 \pi k / 36, k=\{0,1,2,3,4,5,6\}$. The blue line with cusp at $|\psi|=1$ is for $k=\{0,6\}$ (conifold points) and rest of the blue lines are for $k=1,2,3,4,5$ in increasing order of height. As $|\psi| \rightarrow \infty, \arg (\Phi / \Psi) \rightarrow 0$. Clearly, at no point on the moduli space, other than $\psi=\infty, \arg (\Phi / \Psi)=0$ or $\pi$. Or, at no point $\Phi$ and $\Psi$ align, hence, the states with relatively prime $(x, y)$ mentioned above will be stable.

It is important to point out that above we have only considered light states and shown that there are infinitely many light states which are stable against decay into each other. The states which are not of the form that belongs to $\mathcal{Q}$ have divergent mass as we approach $\psi=0$, let's call them massive states. The non-triviality enters when we move away from $\psi=0$ which leads to the light states gaining mass and the massive states becoming light. It turns out that performing calculations similar to above, we can see that the light states can decay into massive ones and vice-versa away from $\psi=0$. 


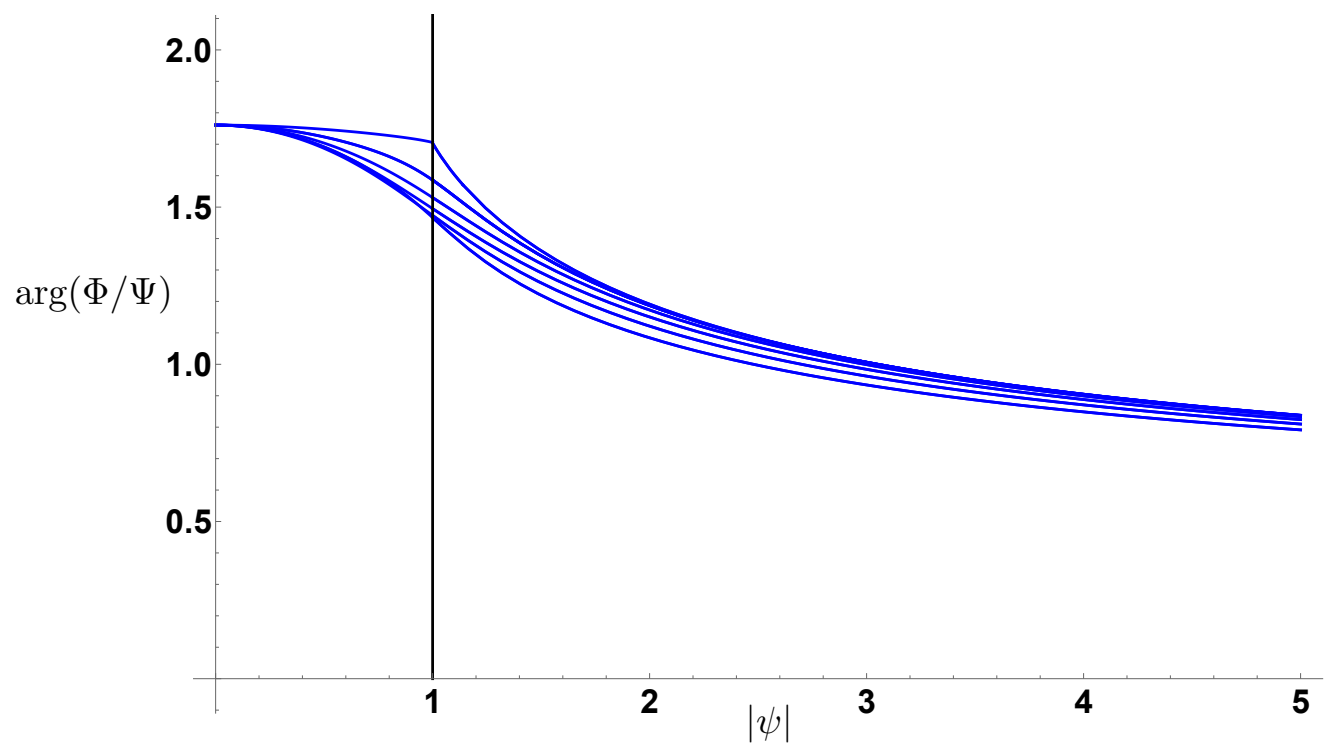

Figure 6. Plot of $\arg (\Phi / \Psi)$ against $|\psi|$ for $\arg (\psi)=2 \pi k / 36, k=\{0,1,2,3,4,5,6\}$. The blue line with cusp at $|\psi|=1$ is for $k=\{0,6\}$ and rest of the blue lines are for $k=1,2,3,4,5$ in increasing order of height.

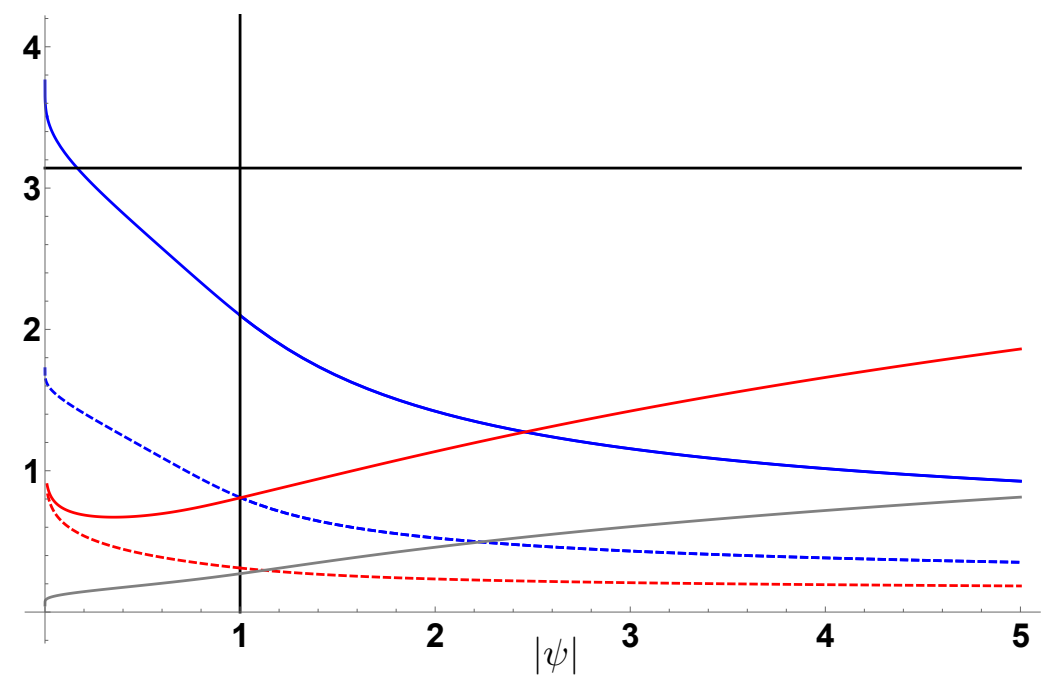

Figure 7. Mass and $\arg (\Phi / \Xi)$ against $|\psi|$ for $\arg (\psi)=2 \pi / 18$. Blue lines show $\arg (\Phi / \Xi)$, red lines show the mass. Dashed is for $\tilde{\mathbf{q}}_{1}=(0,1,0,0)^{\mathrm{T}}$ and thick is for $\tilde{\mathbf{q}}_{2}=(1,1,1,0)^{\mathrm{T}}$. Grey line shows $|\Phi|$.

Let's start with defining

$$
\Xi=e^{K / 2}\left(\tilde{\mathbf{q}}^{\mathrm{T}} \Sigma \Pi\right)
$$

where $\tilde{\mathbf{q}} \notin \mathcal{Q}$. In figure 7 , the plot shows the $\arg (\Phi / \Xi)$ for $\tilde{\mathbf{q}}_{1}=(0,1,0,0)^{\mathrm{T}}$ in dashed blue line and for $\tilde{\mathbf{q}}_{2}=(1,1,1,0)^{\mathrm{T}}$ in thick blue line. Also, in dashed red and thick red, we have plotted $m=|\Xi|$ for $\tilde{\mathbf{q}}_{1}$ and $\tilde{\mathbf{q}}_{2}$ respectively and grey line shows $|\Phi|{ }^{20}$ Note that for $\tilde{\mathbf{q}}_{1}$,

\footnotetext{
${ }^{20} \Phi$ is charge for $\mathbf{q}_{1}=(1,0,4 / 9,1 / 3)^{\mathrm{T}}$, hence $|\Phi|$ represents $1 / 9^{\text {th }}$ of the mass of a state given by $\mathbf{q}=(9,0,4,3)^{\mathrm{T}}$ (quantisation condition).
} 
the blue line crosses the black line $(\arg (\Phi / \Xi)=\pi)$ near $\psi=0$ aligning the two charges whereas for $\tilde{\mathbf{q}}_{2}$ it never happens, also, at this point, the light state and heavy state have comparable mass. Hence, we can conclude that there are lines of marginal stability near $\psi=0$ if all the states are taken into account.

This is however not a problem for us because, in the limit of approaching $\psi=0$, the heavy states become infinitely heavy and light states become massless not allowing any decay to take place between the two classes and the light states remain stable. And hence, the light states with relatively prime $x$ and $y$, under the assumption of stability close to $\psi=0$ advocate for SDC. Also, we have shown that for a state which becomes massive at singularity, there is always a finite radius around the $K$-point in which an infinite number of light states are stable against decay into each other as well as the above mentioned charge.

Next, we will do a similar analysis for $X_{2,2,2,2}$.

\subsection{3 $X_{2,2,2,2}$}

It turns out that for $X_{2,2,2,2}$, everything follows just like it did for $X_{3,3}$. Again, using the period vector calculated in section 4.3 we can find $e^{K / 2}$,

$$
e^{K / 2}=\frac{\sqrt{3} \pi^{3 / 2}}{64|\psi|^{4}(-\log (|\psi|))^{3 / 2}}+\frac{3 \sqrt{3} \pi^{3 / 2} \log (2)}{128|\psi|^{4}(-\log (|\psi|))^{5 / 2}}+\mathcal{O}\left(\frac{1}{|\psi|^{4}(-\log (|\psi|))^{7 / 2}}\right) .
$$

Next, we look at the behaviour of $\mathbf{q}^{\mathrm{T}} \Sigma \Pi$ term in equation (6.14). For general charge $\mathbf{q}$, now the leading (largest) contribution is of order $\psi^{4} \log ^{3}(\psi)$ with sub-leading contributions of order $\psi^{4} \log ^{2}(\psi)$ and $\psi^{4} \log (\psi)$ respectively. In contrast to the case in the last section, we just need to eliminate terms with logarithmic order two or higher to get a convergent, vanishing mass (central charge). We find that this is accomplished using the subset of charge lattice consisting of charges of the form

$$
\mathbf{q}=\left(\begin{array}{c}
x \\
y \\
\frac{x}{2}+\frac{y}{4} \\
\frac{x}{4}
\end{array}\right)
$$

where $x$ and $y$ are arbitrary integers such that $x \bmod 4=0$ and $y \bmod 4=0$ (quantisation condition). We denote this subset by

$$
\mathcal{Q}=\{\mathbf{q} \mid x, y \in \mathbb{Z}, x \bmod 4=0,(2 x+y) \bmod 4=0\} \backslash\left\{(0,0,0,0)^{\mathrm{T}}\right\} .
$$

For these charges, we find

$$
Z_{\mathbf{q}}=e^{K / 2}\left(-\frac{4 i y}{\pi} \psi^{4} \log (\psi)+\frac{2 i y \log (4)-x}{\pi} \psi^{4}+\mathcal{O}\left(\psi^{12}\right)\right) .
$$

Thus, the mass of a BPS state is

$$
m_{\mathbf{q}}=e^{K / 2}\left|\mathbf{q}^{\mathrm{T}} \Sigma \Pi\right|=\frac{\sqrt{3 \pi}|y|}{16 \sqrt{-\log (|\psi|)}}+\mathcal{O}\left(\frac{1}{(-\log (|\psi|))^{3 / 2}}\right) .
$$


Note that the equation above has no $x$ dependence. This doesn't mean that the lattice is one dimensional, it merely means that the $x$ dependence is sub-leading which can be seen from equation (6.32). This would mean that the spacing of mass states is different in the $x$ and $y$ direction. That is, for fixed $x$ and different $y$, we will have larger mass difference than that of different $x$ and fixed $y$.

Therefore, just like in the last section, using the metric from table 4 , we find that the ratio of masses at $Q$ and $P$ for $1 \gg\left|\psi_{P}\right|>\left|\psi_{Q}\right|$ is

$$
\frac{m_{\mathbf{q}}^{Q}}{m_{\mathbf{q}}^{P}} \sim \sqrt{\frac{\log \left(\left|\psi_{P}\right|\right)}{\log \left(\left|\psi_{Q}\right|\right)}} \sim e^{-\frac{1}{\sqrt{3}} d_{P Q}},
$$

as we approach the singularity at $\psi=0$ following the behaviour predicted by SDC.

\subsubsection{Stability of branes in the $X_{2,2,2,2}$ model}

The stability arguments follow analogous to the ones in the last section. We first decompose the central charge as

$$
\begin{aligned}
Z_{\mathbf{q}} & =e^{K / 2}\left(\mathbf{q}^{\mathrm{T}} \Sigma \Pi\right) \\
& =x \Phi+y \Psi
\end{aligned}
$$

where

$$
\begin{gathered}
\Phi=e^{K / 2}\left(\mathbf{q}_{1}^{\mathrm{T}} \Sigma \Pi\right) \text { with } \quad \mathbf{q}_{1}=\left(\begin{array}{c}
1 \\
0 \\
1 / 2 \\
1 / 4
\end{array}\right) \text { and } \\
\Psi=e^{K / 2}\left(\mathbf{q}_{2}^{\mathrm{T}} \Sigma \Pi\right) \text { with } \quad \mathbf{q}_{2}=\left(\begin{array}{c}
0 \\
1 \\
1 / 4 \\
0
\end{array}\right) .
\end{gathered}
$$

In figure 8, we plot $\arg (\Phi / \Psi)$ against $|\psi|$ for $\arg (\psi)=2 \pi k / 48, k=\{0,1,2,3,4,5,6\}$. Again, at not point on the moduli space, except $\psi=\infty, \Phi$ and $\Psi$ align. Hence, for relatively prime $x$ and $y$, the light states are stable everywhere under decaying into each other.

Next, we define

$$
\Xi=e^{K / 2}\left(\tilde{\mathbf{q}}^{\mathrm{T}} \Sigma \Pi\right)
$$

where $\tilde{\mathbf{q}} \notin \mathcal{Q}$. In figure 9 , we have $\arg (\Phi / \Xi)$ for $\tilde{\mathbf{q}}_{1}=(0,1,0,0)^{\mathrm{T}}$ in dashed blue line and for $\tilde{\mathbf{q}}_{2}=(1,1,1,0)^{\mathrm{T}}$ in thick blue line. Respective red lines show the $m=|\Xi|$ and grey line shows $|\Phi|$. Note again, there exists charges with line of marginal stability which can decay light states into massive states (or vice versa).

However, just like before, this is not a problem assuming that there are stable light states near $\psi=0$ with relatively prime $x$ and $y$.

There are two comment in order. Unlike light states for $X_{3,3}$ which have a finite order monodromy around the $K$-point, the light states of the $X_{2,2,2,2}$ model have infinite order 


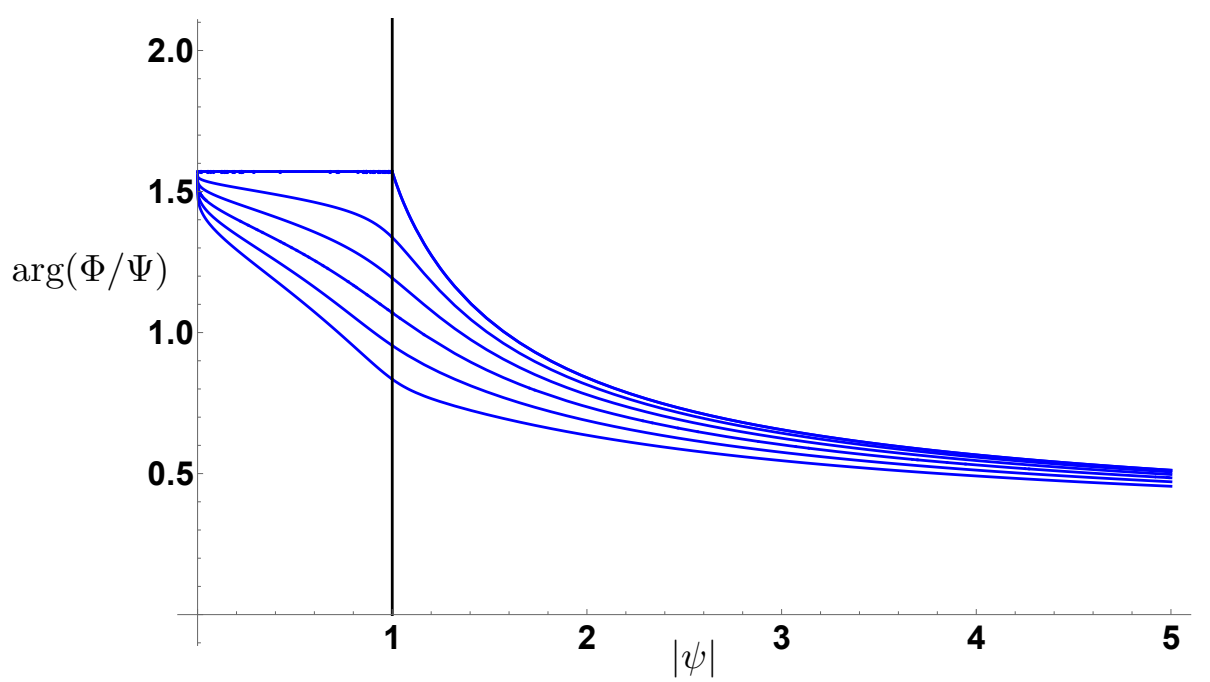

Figure 8. Plot of $\arg (\Phi / \Psi)$ against $|\psi|$ for $\arg (\psi)=2 \pi k / 48, k=\{0,1,2,3,4,5,6\}$. The blue line with cusp at $|\psi|=1$ is for $k=\{0,6\}$ and rest of the blue lines are for $k=1,2,3,4,5$ in increasing order of height.

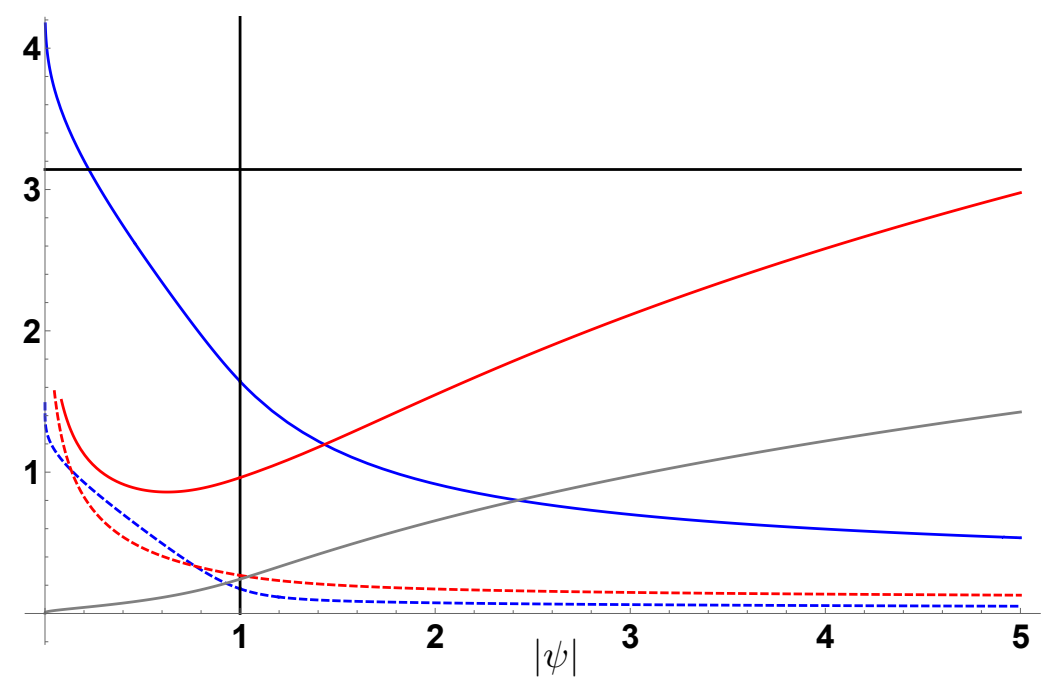

Figure 9. Mass and $\arg (\Phi / \Xi)$ against $|\psi|$ for $\arg (\psi)=2 \pi / 24$. Blue lines show $\arg (\Phi / \Xi)$, red lines show the mass. Dashed is for $\tilde{\mathbf{q}}_{1}=(0,1,0,0)^{\mathrm{T}}$ and thick is for $\tilde{\mathbf{q}}_{2}=(1,1,1,0)^{\mathrm{T}}$. Grey line shows $|\Phi|$.

monodromy so the argument of $[5,13]$ could apply. But there is a much better argument confirming the stability of infinite states including their degenerations. Using the results of [10] one can calculate the $\mathcal{F}_{g}$ at the new $M$-points. As it turns out, even though the leading order of the periods is different, one can make a Kähler transformation so that the genus one prepotential $\mathcal{F}_{0}$ in the flat coordinates $s=Y_{1} / Y_{0}$ is given by the ratio of the single logarithmic by the analytic period becomes exactly the same as the one at the original $M$-point. To see this, we note that the period calculated in section 4.3 near $s$-point 


\begin{tabular}{|l|rrrrrrr|}
\hline $\mathrm{g}$ & $\beta=1$ & $\beta=2$ & $\beta=3$ & $\beta=4$ & $\beta=5$ & $\beta=6$ & $\beta=7$ \\
\hline 0 & 512 & 9728 & 416256 & 25703936 & 1957983744 & 170535923200 & 16300354777600 \\
1 & 0 & 0 & 0 & 14752 & 8782848 & 2672004608 & 615920502784 \\
2 & 0 & 0 & 0 & 0 & 0 & 1427968 & 2440504320 \\
3 & 0 & 0 & 0 & 0 & 0 & 0 & 86016 \\
4 & 0 & 0 & 0 & 0 & 0 & 0 & 0 \\
\hline
\end{tabular}

Table 5. $I_{g}^{\beta}$ for the degree $(2,2,2,2)$ complete intersection in $\mathbb{P}^{7}$ at the second $M$-point $(\psi=0)$.

for $X_{2,2,2,2}$ can be written as

$$
\Pi_{s}(w)=4 \sqrt{\tilde{w}}\left(\begin{array}{cccc}
-4 & 0 & 0 & 0 \\
0 & 4 & 0 & 0 \\
-2 & 1 & -4 & 0 \\
-1 & 0 & 0 & 4
\end{array}\right) \Pi_{M}(\tilde{w})
$$

where $\tilde{w}=2^{16} w$ and $\Pi_{M}$ is from section 4.1. The same is true for the higher genus potentials. Then we can perform the Schwinger loop calculation and determine the BPS indices at the second $M$-points in the same way then at the original $M$-point only that the charges of the light states are not given by the $D 2$ and $D 0$ brane charges at the original $M$-points. However, we can fix the boundary conditions for the $\mathcal{F}_{g}$ so that the BPS indices of the corresponding bound states are exactly the same as the ones at the original $M$-point calculated in [10] and displayed in table 5.

\subsection{Other one parameter hypergeometric systems}

With the help of Meijer G-functions and knowledge of periods around the $M$-point, we find the structure of the metric on approaching the $s$-point for all the 14 hypergeometric one parameter cases and also find the lattice subspace for cases with infinite distance. We present the results in table 1 where we also mention the constants $\mu, \kappa$ and $c_{2} \cdot D$ needed to find the periods around $M$-point (see equation (4.3)). We show the structure of the metric when we approach $s$-point and also the distance to the $s$-point from a non-singular point on the moduli space.

Further, for all examples where the distance to $s$-point in infinite, we can find the subset of charge lattice leading to massless states on approaching the $s$-point. The general form of charge vector for each case is given below.

$$
\begin{aligned}
& X_{2,2,2,2}\left(1^{8}\right): \mathbf{q}=\left(\begin{array}{c}
x \\
y \\
\frac{x}{2}+\frac{y}{4} \\
\frac{x}{4}
\end{array}\right), \\
& x \\
& y \\
& X_{4,4}\left(1^{4} 2^{2}\right): \mathbf{q}=\left(\begin{array}{c}
x, 3\left(1^{6}\right): \mathbf{q}=\left(\begin{array}{c}
x \\
y \\
\frac{4 x}{9}+\frac{y}{3} \\
\frac{x}{3}
\end{array}\right), \\
\frac{x}{2}+\frac{y}{2}
\end{array}\right) \text { and } \quad X_{6,6}\left(1^{2} 2^{2} 3^{2}\right): \mathbf{q}=\left(\begin{array}{c}
x \\
y \\
y \\
x
\end{array}\right) .
\end{aligned}
$$




\section{Acknowledgments}

We would like to thank Hans Jockers and Christoph Nega for useful discussions. We would also like to thank Andreas Gerhardus for proofreading the paper. AJ would like to thank the Bonn-Cologne Graduate School of Physics and Astronomy (BCGS) for their financial support.

\section{A Meijer G-functions}

Here, we briefly describe how one goes about solving the following differential equation

$$
\left(\vartheta^{4}-\mu \frac{1}{w} \prod_{k=1}^{4}\left(\vartheta-a_{k}\right)\right) f(w)=0,
$$

where $\vartheta=w \frac{d}{d w}$. This equation can be rewritten as

$$
\left(\prod_{k=1}^{4}\left(\theta-a_{k}\right)-x \theta^{4}\right) f(x)=0
$$

where $x=\frac{w}{\mu}$ and $\theta=x \frac{d}{d x}$. The differential equation (A.2) is an example of a general differential equation of type

$$
\left(\prod_{k=1}^{q}\left(\theta-b_{k}\right)-x \prod_{k=1}^{p}\left(\theta+1-c_{k}\right)\right) f(x)=0,
$$

whose family of solutions was introduced by Meijer [40] and are denoted by

$$
f(x)=G_{p, q}^{m, n}\left((-1)^{m+n+p} x \mid \begin{array}{l}
c_{1}^{\prime}, \ldots, c_{p}^{\prime} \\
b_{1}^{\prime}, \ldots, b_{q}^{\prime}
\end{array}\right), \quad 0<m \leq q, 0<n \leq p,
$$

where $\left\{c_{k}^{\prime}\right\}$ and $\left\{b_{k}^{\prime}\right\}$ are permutations of the original $\left\{c_{k}\right\}$ and $\left\{b_{k}\right\}$. [41] gives a nice introduction to these functions which are known as Meijer $G$-functions. For our case, on comparing equation (A.2) and (A.3), we find

$$
\begin{aligned}
p & =q=4 \\
c_{k} & =1 \\
b_{k} & =a_{k} .
\end{aligned}
$$

On counting the total number of solutions, we find that there are $4 \times 4$ solutions due to choices of $m$ and $n$, and for each of them, we will have multiple solutions due to the permutations of $\left\{c_{k}\right\}$ and $\left\{b_{k}\right\}$ (or one if all $b_{k}$ are equal).

Note that the differential equation we have is of the fourth order, which means that there should only be four independent solutions. This is consistent with the larger number of solutions above because most of the solutions constructed using Meijer functions are 


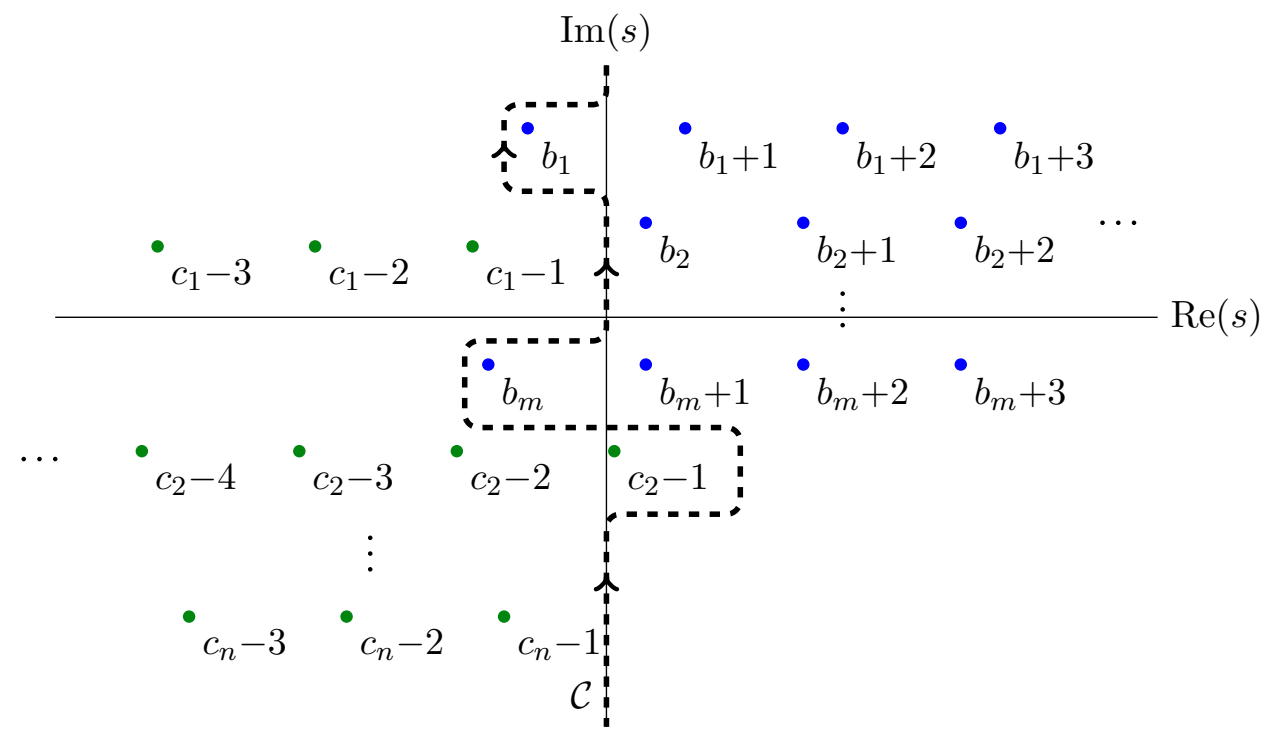

Figure 10. The contour $\mathcal{C}$ with the poles of $\Gamma\left(b_{k}-s\right), k=1, \ldots, m$ marked with blue and the poles of $\Gamma\left(1-c_{k}+s\right), k=1, \ldots, n$ marked with green.

linearly dependent. To see this more clearly, we look at the Mellin-Barnes type integral representation of these functions. We define [42]

$$
G_{p, q}^{m, n}\left(x \mid \begin{array}{l}
c_{1}, \ldots, c_{p} \\
b_{1}, \ldots, b_{q}
\end{array}\right)=\frac{1}{2 \pi i} \int_{\mathcal{C}} \frac{\prod_{k=1}^{m} \Gamma\left(b_{k}-s\right) \prod_{k=1}^{n} \Gamma\left(1-c_{k}+s\right)}{\prod_{k=m+1}^{q} \Gamma\left(1-b_{k}+s\right) \prod_{k=n+1}^{p} \Gamma\left(c_{k}-s\right)} x^{s} d s
$$

Here, empty product is taken to be 1 . The contour $\mathcal{C}$ is shown in figure 10. Here, with blue, we have marked the poles of $\Gamma\left(b_{k}-s\right), k=1, \ldots, m$ and with green, we have poles of $\Gamma\left(1-c_{k}+s\right), k=1, \ldots, n$. There are two ways we can close this contour:

1. $\mathcal{C}$ is closed to the right encircling all of the poles of $\Gamma\left(b_{k}-s\right), k=1, \ldots, m$, once in the negative direction and none of the poles of $\Gamma\left(1-c_{k}+s\right), k=1, \ldots, n$. This will converge for $|x|<1$.

2. $\mathcal{C}$ is closed to the left encircling all of the poles of $\Gamma\left(1-c_{k}+s\right), k=1, \ldots, n$, once in the positive direction and none of the poles of $\Gamma\left(b_{k}-s\right), k=1, \ldots, n$. This will converge for $|x|>1$.

The following are some useful observations. The family of solutions we had before, equation (A.4), will have redundancies since we can commute Gamma functions in the numerator or in the denominator of right side of the equation (A.6). The integral representation is also useful in analytic continuation of the function from a region where $|x|<1$ to a region where $|x|>1$ or vice versa by closing the contour on different sides.

For $p=q$, if no two $b_{j}, j=1, \ldots, m$ differ by an integer, then all poles of (A.6) will be of first order if we close the contour to the right, i.e. for $|x|<1$, which allows us to write 
the Meijer functions in terms of hypergeometric functions ${ }_{a} F_{b}[42]$

$$
\begin{aligned}
& G_{p, q}^{m, n}\left(x \mid \begin{array}{l}
c_{1}, \ldots, c_{p} \\
b_{1}, \ldots, b_{q}
\end{array}\right)= \sum_{h=1}^{m} \frac{\prod_{j=1}^{m} \Gamma\left(b_{j}-b_{h}\right) \prod_{j=1}^{n} \Gamma\left(1+b_{h}-c_{j}\right)}{\prod_{j=m+1}^{q} \Gamma\left(1+b_{h}-b_{j}\right) \prod_{j=n+1}^{p} \Gamma\left(c_{j}-b_{h}\right)} x^{b_{h}} \\
& \times{ }_{p} F_{q-1}\left(1+b_{h}-c_{1}, \ldots, 1+b_{h}-c_{p} ;\right. \\
&\left.1+b_{h}-b_{1}, \ldots, *, \ldots, 1+b_{h}-b_{q} ;(-1)^{p-m-n} x\right),
\end{aligned}
$$

where prime in $\Pi^{\prime}$ indicates that we omit term $\Gamma\left(b_{h}-b_{h}\right)$ and the asterisk indicates omission of $1+b_{h}-b_{h}$ term. The hypergeometric functions can be written as a power series

$$
{ }_{u} F_{v}\left(a_{1}, \ldots, a_{u} ; b_{1}, \ldots, b_{v} ; x\right)=\sum_{n=0}^{\infty} \frac{\left(a_{1}\right)_{n} \ldots\left(a_{u}\right)_{n}}{\left(b_{1}\right)_{n} \ldots\left(b_{v}\right)_{n}} \frac{x^{n}}{n !},
$$

where

$$
(a)_{k}=\frac{\Gamma(a+k)}{\Gamma(a)}, \quad(a)_{0}=1 .
$$

Let us now use the method stated above to explicitly find the solutions of equation (A.1) for the cases discussed in this work. Following are the results $\left(x=\frac{w}{\mu}\right)$ :

1. For $X_{4,2}\left(1^{6}\right)$, we have $\left(a_{1}, a_{2}, a_{3}, a_{4}\right)=(1 / 4,1 / 2,1 / 2,3 / 4)$ and $\mu=2^{10}$.

Family of solutions:

$$
G_{4,4}^{m, n}\left(\left.(-1)^{m+n+4} \frac{w}{2^{10}}\right|_{\frac{1}{4}^{\prime}, \frac{1}{2}^{\prime}, \frac{1}{2}^{\prime}, \frac{3}{4}^{\prime}} ^{1,1}\right) .
$$

Four linearly independent solutions:

$$
\begin{aligned}
& f_{1}(w)=G_{4,4}^{1,4}\left(-\frac{w}{2^{10}} \mid \begin{array}{c}
1,1,1,1 \\
\frac{1}{4}, \frac{1}{2}, \frac{1}{2}, \frac{3}{4}
\end{array}\right) \\
& \stackrel{|w|<2^{10}}{\longrightarrow} \frac{\Gamma\left(\frac{1}{4}\right)^{4}}{\sqrt{\pi} \Gamma\left(\frac{3}{4}\right)^{2}}\left(\frac{-w}{2^{10}}\right)^{1 / 4}{ }_{4} F_{3}\left(\frac{1}{4}, \frac{1}{4}, \frac{1}{4}, \frac{1}{4} ; \frac{1}{2}, \frac{3}{4}, \frac{3}{4} ; \frac{w}{2^{10}}\right), \\
& f_{2}(w)=G_{4,4}^{1,4}\left(-\frac{w}{2^{10}} \mid \frac{1}{\frac{1}{2}}, \frac{1}{4}, \frac{1}{2}, \frac{3}{4}\right)
\end{aligned}
$$


2. For $X_{3,3}\left(1^{6}\right)$, we have $\left(a_{1}, a_{2}, a_{3}, a_{4}\right)=(1 / 3,1 / 3,2 / 3,2 / 3)$ and $\mu=3^{6}$.

Family of solutions:

$$
G_{4,4}^{m, n}\left(\left.(-1)^{m+n+4} \frac{w}{3^{6}}\right|_{\frac{1}{3}^{\prime}, \frac{1}{3}^{\prime}, \frac{2}{3}^{\prime}, \frac{2}{3}^{\prime}} ^{1,1,1,1}\right) .
$$

Four linearly independent solutions:

$$
\begin{aligned}
& f_{1}(w)=G_{4,4}^{1,4}\left(-\frac{w}{3^{6}} \mid \begin{array}{l}
1,1,1,1 \\
\frac{1}{3}, \frac{1}{3}, \frac{2}{3}, \frac{2}{3}
\end{array}\right) \\
& \stackrel{|w|<3^{6}}{\longrightarrow} \frac{\Gamma\left(\frac{1}{3}\right)^{4}}{\Gamma\left(\frac{2}{3}\right)^{2} \Gamma(1)}\left(-\frac{w}{3^{6}}\right)^{1 / 3}{ }_{4} F_{3}\left(\frac{1}{3}, \frac{1}{3}, \frac{1}{3}, \frac{1}{3} ; \frac{2}{3}, \frac{2}{3}, 1 ; \frac{w}{3^{6}}\right), \\
& f_{2}(w)=G_{4,4}^{1,4}\left(-\left.\frac{w}{3^{6}}\right|_{\frac{2}{3}, \frac{1}{3}, \frac{1}{3}, \frac{2}{3}} ^{1,1,1,1}\right) \\
& \stackrel{|w|<3^{6}}{\longrightarrow} \frac{\Gamma\left(\frac{2}{3}\right)^{4}}{\Gamma(1) \Gamma\left(\frac{4}{3}\right)^{2}}\left(-\frac{w}{3^{6}}\right)^{2 / 3}{ }_{4} F_{3}\left(\frac{2}{3}, \frac{2}{3}, \frac{2}{3}, \frac{2}{3} ; 1, \frac{4}{3}, \frac{4}{3} ; \frac{w}{3^{6}}\right), \\
& f_{3}(w)=G_{4,4}^{2,4}\left(\left.\frac{w}{3^{6}}\right|_{\frac{1}{3}, \frac{1}{3}, \frac{2}{3}, \frac{2}{3}} ^{1,1,1,1}\right), \\
& f_{4}(w)=G_{4,4}^{2,4}\left(\frac{w}{3^{6}} \mid \begin{array}{l}
1,1,1,1 \\
\frac{2}{3}, \frac{2}{3}, \frac{1}{3}, \frac{1}{3}
\end{array}\right) \text {. }
\end{aligned}
$$

3. For $X_{2,2,2,2}\left(1^{8}\right)$, we have $\left(a_{1}, a_{2}, a_{3}, a_{4}\right)=(1 / 2,1 / 2,1 / 2,1 / 2)$ and $\mu=2^{8}$.

Family of solutions:

$$
G_{4,4}^{m, n}\left(\left.(-1)^{m+n+4} \frac{w}{2^{8}}\right|_{\frac{1}{2}, \frac{1}{2}, \frac{1}{2}, \frac{1}{2}} ^{1,1,1,1}\right)
$$

Four linearly independent solutions:

$$
\begin{aligned}
& f_{1}(w)= G_{4,4}^{1,4}\left(-\frac{w}{2^{8}} \mid \frac{1}{\frac{1}{2}, \frac{1}{2}, \frac{1}{2}, \frac{1}{2}}\right) \\
& \stackrel{|w|<2^{8}}{\longrightarrow} \frac{\Gamma\left(\frac{1}{2}\right)^{4}}{\Gamma(1)^{3}}\left(-\frac{w}{2^{8}}\right)^{1 / 2}{ }_{4} F_{3}\left(\frac{1}{2}, \frac{1}{2}, \frac{1}{2}, \frac{1}{2} ; 1,1,1 ; \frac{w}{2^{8}}\right), \\
& f_{2}(w)=G_{4,4}^{2,4}\left(\frac{w}{2^{8}} \mid \begin{array}{c}
1,1,1,1 \\
\frac{1}{2}, \frac{1}{2}, \frac{1}{2}, \frac{1}{2}
\end{array}\right), \\
& f_{3}(w)=G_{4,4}^{3,4}\left(-\frac{w}{2^{8}} \mid \begin{array}{c}
1,1,1,1 \\
\frac{1}{2}, \frac{1}{2}, \frac{1}{2}, \frac{1}{2}
\end{array}\right) \\
& f_{4}(w)=G_{4,4}^{4,4}\left(\frac{w}{2^{8}} \mid \begin{array}{c}
1,1,1,1 \\
\frac{1}{2}, \frac{1}{2}, \frac{1}{2}, \frac{1}{2}
\end{array}\right) .
\end{aligned}
$$


Open Access. This article is distributed under the terms of the Creative Commons Attribution License (CC-BY 4.0), which permits any use, distribution and reproduction in any medium, provided the original author(s) and source are credited.

\section{References}

[1] H. Ooguri and C. Vafa, On the geometry of the string landscape and the swampland, Nucl. Phys. B 766 (2007) 21 [hep-th/0605264] [INSPIRE].

[2] D. Klaewer and E. Palti, Super-Planckian spatial field variations and quantum gravity, JHEP 01 (2017) 088 [arXiv:1610.00010] [INSPIRE].

[3] D. van Straten, Calabi-Yau operators, in Uniformization, Riemann-Hilbert correspondence, Calabi-Yau manifolds $\&$ Picard-Fuchs equations, L. Ji et al. eds., Advanced Lecture in Mathematics volume 42, Int. Press, Somerville, U.S.A. (2018).

[4] G. Almkvist and D. von Straten, Calabi-Yau differential operator database v.3.

[5] T.W. Grimm, C. Li and E. Palti, Infinite distance networks in field space and charge orbits, JHEP 03 (2019) 016 [arXiv:1811.02571] [INSPIRE].

[6] A. Klemm, P. Mayr and C. Vafa, BPS states of exceptional noncritical strings, Nucl. Phys. Proc. Suppl. 58 (1997) 177 [hep-th/9607139] [INSPIRE].

[7] S.-J. Lee, W. Lerche and T. Weigand, Tensionless Strings and the Weak Gravity Conjecture, JHEP 10 (2018) 164 [arXiv:1808.05958] [INSPIRE].

[8] A. Klemm et al., Selfdual strings and $N=2$ supersymmetric field theory, Nucl. Phys. B 477 (1996) 746 [hep-th/9604034] [INSPIRE].

[9] N. Seiberg and E. Witten, Electric-magnetic duality, monopole condensation and confinement in $N=2$ supersymmetric Yang-Mills theory, Nucl. Phys. B 426 (1994) 19 [Erratum ibid. B 430 (1994) 485] [hep-th/9407087] [INSPIRE].

[10] M.X. Huang, A. Klemm and S. Quackenbush, Topological string theory on compact Calabi-Yau: modularity and boundary conditions, Lect. Notes Phys. 757 (2009) 45 [hep-th/0612125] [INSPIRE].

[11] P. Corvilain, T.W. Grimm and I. Valenzuela, The swampland distance conjecture for Kähler moduli, arXiv: 1812.07548 [INSPIRE].

[12] R. Blumenhagen, D. Kläwer, L. Schlechter and F. Wolf, The refined swampland distance conjecture in Calabi-Yau moduli spaces, JHEP 06 (2018) 052 [arXiv: 1803. 04989] [INSPIRE].

[13] T.W. Grimm, E. Palti and I. Valenzuela, Infinite distances in field space and massless towers of states, JHEP 08 (2018) 143 [arXiv:1802.08264] [INSPIRE].

[14] T. Banks and N. Seiberg, Symmetries and strings in field theory and gravity, Phys. Rev. D 83 (2011) 084019 [arXiv: 1011.5120] [INSPIRE].

[15] P. Candelas and X.C. de la Ossa, Moduli space of Calabi-Yau manifolds, Nucl. Phys. B 355 (1991) 455.

[16] C. F. Doran and J. W. Morgan, Mirror symmetry and integral variations of Hodge structure underlying one-parameter families of Calabi-Yau threefolds, in Mirror symmetry $V$, N. Yui et al eds., AMS/IP Studies in Advanced Mathematics volume 38, American Mathematical Society, U.S.A. (2006). 
[17] D.R. Morrison, Picard-Fuchs equations and mirror maps for hypersurfaces, in Essays on mirror manifolds, S.T. Yau ed., Int. Press, Somerville, U.S.A. (1992).

[18] A. Klemm and S. Theisen, Considerations of one modulus Calabi-Yau compactifications: Picard-Fuchs equations, Kähler potentials and mirror maps, Nucl. Phys. B 389 (1993) 153 [hep-th/9205041] [INSPIRE].

[19] A. Font, Periods and duality symmetries in Calabi-Yau compactifications, Nucl. Phys. B 391 (1993) 358 [hep-th/9203084] [INSPIRE].

[20] A. Libgober and J. Teitelbaum, Lines on Calabi-Yau complete intersections, mirror symmetry, and Picard-Fuchs equations, Int. Math. Res. Not. 1 (1993) 29.

[21] A. Klemm and S. Theisen, Mirror maps and instanton sums for complete intersections in weighted projective space, Mod. Phys. Lett. A 9 (1994) 1807 [hep-th/9304034] [INSPIRE].

[22] D.A. Cox and S. Katz, Mirror symmetry and algebraic geometry, Mathematical Surveys and Monographs volume 68, American Mathematical Society, U.S.A. (1999).

[23] S. Hosono, A. Klemm, S. Theisen and S.-T. Yau, Mirror symmetry, mirror map and applications to complete intersection Calabi-Yau spaces, Nucl. Phys. B 433 (1995) 501 [hep-th/9406055] [INSPIRE].

[24] W. Schmid, Variation of Hodge structure: the singularities of the period mapping, Invent. Math. 22 (1973) 211.

[25] C.L. Wang, On the incompleteness of the weil-petersson metric along degenerations of calabi-yau manifolds, Math. Res. Lett. 4 (1997) 157.

[26] B. Greene and M. Plesser, Duality in Calabi-Yau moduli space, Nucl. Phys. B 338 (1990) 15.

[27] A. Libgober and J. Teitelbaum, Lines on Calabi-Yau complete intersections, mirror symmetry and Picard-Fuchs equations, alg-geom/9301001 [INSPIRE].

[28] P. Berglund et al., Periods for Calabi-Yau and Landau-Ginzburg vacua, Nucl. Phys. B 419 (1994) 352.

[29] A. Klemm, E. Scheidegger and D. Zagier, Periods and quasiperiods of modular forms and d-brane masses for the mirror quintic, in preparation.

[30] I. Antoniadis, E. Gava, K.S. Narain and T.R. Taylor, Topological amplitudes in string theory, Nucl. Phys. B 413 (1994) 162 [hep-th/9307158] [InSPIRE].

[31] R. Gopakumar and C. Vafa, M theory and topological strings. 2., hep-th/9812127 [INSPIRE].

[32] S.H. Katz, A. Klemm and C. Vafa, M theory, topological strings and spinning black holes, Adv. Theor. Math. Phys. 3 (1999) 1445 [hep-th/9910181] [InSPIRE].

[33] R. Pandharipande and R.P. Thomas, Stable pairs and BPS invariants, J. Amer. Math. Soc. 23 (2010) 267.

[34] A. Strominger, Massless black holes and conifolds in string theory, Nucl. Phys. B 451 (1995) 96 [hep-th/9504090] [INSPIRE].

[35] M.-x. Huang, A. Klemm, M. Mariño and A. Tavanfar, Black holes and large order quantum geometry, Phys. Rev. D 79 (2009) 066001 [arXiv:0704.2440] [InSPIRE].

[36] C. Vafa, A stringy test of the fate of the conifold, Nucl. Phys. B 447 (1995) 252 [hep-th/9505023] [INSPIRE]. 
[37] B.R. Greene, String theory on Calabi-Yau manifolds, in the proceedings of Fields, strings and duality. Summer School, Theoretical Advanced Study Institute in Elementary Particle Physics (TASI'96), June 2-28, Boulder, U.S.A. (1996), hep-th/9702155 [INSPIRE].

[38] P. Candelas, X.C. De La Ossa, P.S. Green and L. Parkes, A pair of Calabi-Yau manifolds as an exactly soluble superconformal theory, Nucl. Phys. B 359 (1991) 21 [InSPIRE].

[39] A. Ceresole, R. D'Auria, S. Ferrara and A. Van Proeyen, Duality transformations in supersymmetric Yang-Mills theories coupled to supergravity, Nucl. Phys. B 444 (1995) 92 [hep-th/9502072] [INSPIRE].

[40] C.S. Meijer, Über Whittakersche bzw. Besselsche Funktionen und deren Produkte, Nieuw Arch. Wiskd. II. Ser. 18 (1936) 10.

[41] R. Beals and J. Szmigielski, Meijer g-functions: a gentle introduction, Not. AMS 60 (2013) 866.

[42] H. Bateman, Higher transcendental functions. Volume I, McGraw-Hill, U.S.A. (1953). 